\title{
Development and Application of a New General Method for the Asymmetric Synthesis of syn- and anti-1,3-Amino Alcohols.
}

\author{
Takuya Kochi, Tony P. Tang, Jonathan A. Ellman* \\ Center for New Directions in Organic Synthesis, Department of \\ Chemistry, University of California, Berkeley, California 94720, USA
}

\section{Experimental Section}

General Methods. Unless otherwise noted, all reagents were obtained from commercial suppliers and were used without further purification. $\mathrm{MgBr}_{2}$ and $\mathrm{ZnBr}_{2}$ were purchased from Aldrich. Tetrahydrofuran (THF) and diethyl ether $\left(\mathrm{Et}_{2} \mathrm{O}\right)$ were distilled from $\mathrm{Na}$ /benzophenone ketyl. Diisopropylamine $\left(i-\mathrm{Pr}_{2} \mathrm{NH}\right)$ was distilled from $\mathrm{CaH}_{2}$. Toluene was distilled from Na. All aldehydes and ketones were distilled before use, except for isobutyraldehyde, which was obtained in a Sure/Seal ${ }^{\mathrm{TM}}$ bottle from Aldrich. The concentration of commercially available solutions of $n$-butyllithium in pentane was determined by titration with 2,6-di-tert-butyl-4-methylphenol and 1,10-phenanthroline. All reactions were carried out under a $\mathrm{N}_{2}$ atmosphere in flame- or oven-dried glassware. Unless otherwise noted, extracts were dried over $\mathrm{Na}_{2} \mathrm{SO}_{4}$. Chromatography was carried out with Merck 60 230-400 mesh silica gel according to the procedure described by Still. ${ }^{1}$ Reactions and chromatography fractions were analyzed with thin layer chromatography on Merck $60 \mathrm{~F}_{254} 0.25$ micron silica plates. IR spectra were recorded on a Nicolet Avatar 360 FTIR spectrometer equipped with an attenuated total reflectance accessory and only partial data are listed. ${ }^{1} \mathrm{H}$ and ${ }^{13} \mathrm{C} \mathrm{NMR}$ spectra were obtained in $\mathrm{CDCl}_{3}$ at room temperature. Chemical shifts in NMR spectra are expressed in ppm relative to $\mathrm{CDCl}_{3}$. Coupling constants are reported in $\mathrm{Hz}$. For $\mathbf{2 n - q}$, only the chemical shifts of the major isomer were listed. Unless otherwise noted, HPLC analysis was performed with a $25 \mathrm{~cm}$ x $5 \mu \mathrm{m}$ ID Microsorb Si normal phase column with UV detection at $280 \mathrm{~nm}$ and at $254 \mathrm{~nm}$. Elemental analyses and mass spectra were obtained from the Microanalytical Laboratory at the University of California, Berkeley. tert-Butanesulfinyl imines $\mathbf{1}$ were prepared as previously described. ${ }^{2}$ Ketone 7 was prepared by the method of Grigg and co-workers. ${ }^{3}$

General Procedure A for the Addition of Metalloenamine Derived from tert-Butanesulfinyl Imine $(\boldsymbol{R})-1$ to Aldehydes. To a $0.240 \mathrm{M}$ solution of $i$ - $\operatorname{Pr}_{2} \mathrm{NH}(1.20$ equiv) in THF was added butyllithium in pentane (1.10 equiv) at $0{ }^{\circ} \mathrm{C}$ and the mixture was stirred for $30 \mathrm{~min}$. The solution was then cooled to $-78{ }^{\circ} \mathrm{C}$ and tert-butanesulfinyl imine (R)-1 (1.00 equiv) was added. After the reaction solution was stirred for $45 \mathrm{~min}$ at 
$-78{ }^{\circ} \mathrm{C}$, the additive (2.00 equiv) was added. The reaction mixture was stirred for 45 min, and the aldehyde (1.10-1.30 equiv) was added to the solution. Then, the mixture was stirred for $1-3 \mathrm{~h}$ at $-78{ }^{\circ} \mathrm{C}$. To the reaction mixture was added $2 \mathrm{~N}$ AcOH/THF $(5$ equiv), which was cooled to $-78{ }^{\circ} \mathrm{C}$ before the addition. Brine and/or saturated aqueous $\mathrm{NaHCO}_{3}$ solution was then added to the mixture with stirring. The organic layer was collected and the brine layer was extracted with EtOAc (2x). The combined organic portions were quickly dried, filtered, and concentrated. The addition products were purified by silica gel chromatography.

$\beta$-Hydroxy- $N$-Sulfinyl Imine 2a. General procedure A was followed with 223

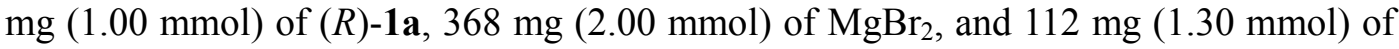
trimethylacetaldehyde. After the addition of the aldehyde, the reaction mixture was stirred for $3 \mathrm{~h}$ before the addition of $2 \mathrm{~N}$ AcOH/THF. Diastereoselectivity was determined by HPLC analysis; 98:2 dr (98:2 hexanes/i-PrOH, $1.0 \mathrm{~mL} / \mathrm{min}$ : major diastereomer, $t_{R}=6.1 \mathrm{~min}$; minor diastereomer, $t_{R}=9.0 \mathrm{~min}$ ). Column chromatography (70:30 hexanes/EtOAc) afforded $198 \mathrm{mg}(64 \%)$ of 2a: IR 1568, 1590, 1602, $3311 \mathrm{~cm}^{-1}$; ${ }^{1} \mathrm{H}$ NMR (400 MHz) $\delta 1.00$ (s, 9H), 1.38 (s, 9H), 3.27 (d, 1H, $J=9.4$ ), 3.31-3.47 (m, 2H), 4.60 (apparent br d, 1H), 7.40-7.52 (m, 3H), 7.84 (d, 2H, $J=7.0$ ); ${ }^{13} \mathrm{C}$ NMR (100 MHz) $\delta 22.9,25.7,35.5,36.1,59.0,75.7,127.5,128.6,131.7,137.4$, 177.6. Anal. Calcd. for $\mathrm{C}_{17} \mathrm{H}_{27} \mathrm{NO}_{2} \mathrm{~S}$ : C, 65.98; H, 8.79; N, 4.53. Found: C, 66.33; H, $8.92 ; \mathrm{N}, 4.42$.

$\beta$-Hydroxy- $\boldsymbol{N}$-Sulfinyl Imine $2 \mathrm{~b}$. General procedure A was followed with 1.12 $\mathrm{g}(5.01 \mathrm{mmol})$ of $(R)-1 \mathrm{a}, 1.84 \mathrm{~g}(10.0 \mathrm{mmol})$ of $\mathrm{MgBr}_{2}$, and $469 \mathrm{mg}(6.50 \mathrm{mmol})$ of isobutyraldehyde. After the addition of the aldehyde, the reaction mixture was stirred for $3 \mathrm{~h}$ before the addition of $2 \mathrm{~N} \mathrm{AcOH} / \mathrm{THF}$. Diastereoselectivity was determined by HPLC analysis; 98:2 dr (98:2 hexanes/ethanol, $1.0 \mathrm{~mL} / \mathrm{min}$ : major diastereomer, $\mathrm{t}_{\mathrm{R}}=$ $11.6 \mathrm{~min}$; minor diastereomer, $\left.t_{\mathrm{R}}=21.1 \mathrm{~min}\right)$. Column chromatography $(80: 20$ hexanes/EtOAc) afforded $1.30 \mathrm{~g}(88 \%)$ of $\mathbf{2 b}$ : IR 1567, 1590, $1601 \mathrm{~cm}^{-1}$; ${ }^{1} \mathrm{H}$ NMR (400 MHz) $\delta 1.00(\mathrm{~d}, 3 \mathrm{H}, J=6.7), 1.01(\mathrm{~d}, 3 \mathrm{H}, J=6.7), 1.37(\mathrm{~s}, 9 \mathrm{H}), 1.74-1.82(\mathrm{~m}, 1 \mathrm{H})$, $3.21(\mathrm{dd}, 1 \mathrm{H}, J=12.4,2.7), 3.42(\mathrm{dd}, 1 \mathrm{H}, J=12.4,11.2), 3.51$ (br s, 1H), 4.54 (apparent br d, $1 \mathrm{H}), 7.43(\mathrm{t}, 2 \mathrm{H}, J=7.3), 7.49(\mathrm{t}, 1 \mathrm{H}, J=7.3), 7.84(\mathrm{~d}, 2 \mathrm{H}, J=7.3) ;{ }^{13} \mathrm{C} \mathrm{NMR}$ (100 MHz) $\delta 17.8,18.9,23.1,35.7,38.2,59.2,73.0,127.7,128.9,132.0,137.6,177.4$. Anal. Calcd. for $\mathrm{C}_{16} \mathrm{H}_{25} \mathrm{NO}_{2} \mathrm{~S}$ : C, 65.05; H, 8.53; N, 4.74. Found: C, 65.18; H, 8.55; N, 4.56 .

$\beta$-Hydroxy- $\boldsymbol{N}$-Sulfinyl Imine 2c. General procedure A was followed with 223 $\mathrm{mg}(1.00 \mathrm{mmol})$ of $(R)-\mathbf{1 a}, 368 \mathrm{mg}(2.00 \mathrm{mmol})$ of $\mathrm{MgBr}_{2}$, and $112 \mathrm{mg}(1.30 \mathrm{mmol})$ of isovaleraldehyde. After the addition of the aldehyde, the reaction mixture was stirred for 
$3 \mathrm{~h}$ before the addition of $2 \mathrm{~N} \mathrm{AcOH} / \mathrm{THF}$. Diastereoselectivity was determined by HPLC analysis; 96:4 dr (97:3 hexanes $/ i-\mathrm{PrOH}, 1.0 \mathrm{~mL} / \mathrm{min}$ : major diastereomer, $\mathrm{t}_{\mathrm{R}}=$ $14.9 \mathrm{~min}$; minor diastereomer, $\left.t_{\mathrm{R}}=30.7 \mathrm{~min}\right)$. Column chromatography (80:20 hexanes/EtOAc) afforded $232 \mathrm{mg}(75 \%)$ of $2 \mathrm{c}$ : IR 1567, 1590, $3319 \mathrm{~cm}^{-1} ;{ }^{1} \mathrm{H}$ NMR (400 MHz) $\delta 0.90(\mathrm{~d}, 3 \mathrm{H}, J=6.6), 0.94(\mathrm{~d}, 3 \mathrm{H}, J=6.7), 1.29-1.41(\mathrm{~m}, 1 \mathrm{H}), 1.36(\mathrm{~s}, 9 \mathrm{H})$, $1.52-1.60(\mathrm{~m}, 1 \mathrm{H}), 1.84-1.94(\mathrm{~m}, 1 \mathrm{H}), 3.22(\mathrm{dd}, 1 \mathrm{H}, J=12.8,3.5), 3.36(\mathrm{dd}, 1 \mathrm{H}, J=$ 12.8, 10.6), 3.82 (br s, 1H), 4.31 (apparent br d, 1H), 7.44 (t, 2H, $J=7.4), 7.50$ (t, 1H, $J$ = 7.4), $7.85(\mathrm{~d}, 2 \mathrm{H}, J=7.4) ;{ }^{13} \mathrm{C}$ NMR (125 MHz) $\delta 22.1,22.8,23.4,24.7,41.7,48.7$, 58.8, 66.4, 127.5, 128.6, 131.8, 137.5, 176.7. Anal. Calcd. for $\mathrm{C}_{17} \mathrm{H}_{27} \mathrm{NO}_{2} \mathrm{~S}: \mathrm{C}, 65.98$; H, 8.79; N, 4.53. Found: C, 66.09; H, 9.02; N, 4.36.

$\boldsymbol{\beta}$-Hydroxy- $\boldsymbol{N}$-Sulfinyl Imine 2d. General procedure A was followed with 223 $\mathrm{mg}(1.00 \mathrm{mmol})$ of $(R)-1 \mathrm{a}, 368 \mathrm{mg}(2.00 \mathrm{mmol})$ of $\mathrm{MgBr}_{2}$, and $75.0 \mathrm{mg}(1.30 \mathrm{mmol})$ of propionaldehyde. After the addition of the aldehyde, the reaction mixture was stirred for $3 \mathrm{~h}$ before the addition of $2 \mathrm{~N} \mathrm{AcOH} / \mathrm{THF}$. Diastereoselectivity was determined by HPLC analysis; 96:4 dr (97:3 hexanes $/ i-\mathrm{PrOH}, 1.0 \mathrm{~mL} / \mathrm{min}$ : major diastereomer, $\mathrm{t}_{\mathrm{R}}=$ $10.1 \mathrm{~min}$; minor diastereomer, $\left.t_{R}=16.2 \mathrm{~min}\right)$. Column chromatography $(70: 30$ hexanes/EtOAc) afforded $236 \mathrm{mg}(84 \%)$ of $2 d$ : IR 1562, 1588, $3319 \mathrm{~cm}^{-1}$; ${ }^{1} \mathrm{H}$ NMR $(400 \mathrm{MHz}) \delta 1.02(\mathrm{t}, 3 \mathrm{H}, J=7.4), 1.37(\mathrm{~s}, 9 \mathrm{H}), 1.61-1.66(\mathrm{~m}, 2 \mathrm{H}), 3.25(\mathrm{dd}, 1 \mathrm{H}, J=$ 12.8, 3.3), 3.39 (dd, $1 \mathrm{H}, J=12.8,10.8$ ), 3.69 (br s, 1H), 4.43 (apparent br d, 1H), 7.43 (t, $2 \mathrm{H}, J=7.4), 7.50(\mathrm{t}, 1 \mathrm{H}, J=7.4), 7.85(\mathrm{~d}, 2 \mathrm{H}, J=7.4) ;{ }^{13} \mathrm{C}$ NMR $(100 \mathrm{MHz}) \delta 9.9$, 22.9, 32.1, 40.7, 58.9, 69.5, 127.5, 128.6, 131.7, 137.4, 176.7. Anal. Calcd. for $\mathrm{C}_{15} \mathrm{H}_{23} \mathrm{NO}_{2} \mathrm{~S}: \mathrm{C}, 64.02 ; \mathrm{H}, 8.24 ; \mathrm{N}, 4.98$. Found: $\mathrm{C}, 63.76 ; \mathrm{H}, 7.98 ; \mathrm{N}, 4.99$.

$\boldsymbol{\beta}$-Hydroxy- $\boldsymbol{N}$-Sulfinyl Imine 2e. General procedure A was followed with $446 \mathrm{mg}(2.00 \mathrm{mmol})$ of $(R)-\mathbf{1 a}, 901 \mathrm{mg}$ (4.00 mmol) of $\mathrm{ZnBr}_{2}$, and $276 \mathrm{mg}(1.30 \mathrm{mmol})$ of benzaldehyde. After the addition of the aldehyde, the reaction mixture was stirred for $3 \mathrm{~h}$ before the addition of $2 \mathrm{~N} \mathrm{AcOH} / \mathrm{THF}$. Diastereoselectivity was determined by HPLC analysis; 92:8 dr (97:3 hexanes $/ i-\mathrm{PrOH}, 1.0 \mathrm{~mL} / \mathrm{min}$ : major diastereomer, $\mathrm{t}_{\mathrm{R}}=$ $8.0 \mathrm{~min}$; minor diastereomer, $\left.t_{\mathrm{R}}=9.8 \mathrm{~min}\right)$. Column chromatography (70:30 hexanes/EtOAc) afforded $519 \mathrm{mg}(79 \%)$ of 2e: IR 1572, 1592, 1604, $3286 \mathrm{~cm}^{-1} ;{ }^{1} \mathrm{H}$ NMR (400 MHz) $\delta 1.43(\mathrm{~s}, 9 \mathrm{H}), 3.46(\mathrm{dd}, 1 \mathrm{H}, J=13.1,3.4), 3.70(\mathrm{dd}, 1 \mathrm{H}, J=13.1$, 11.3), 4.79-4.91 (m, 1H), 5.61 (apparent br d, 1H), 7.27-7.57 (m, 8H), $7.96(\mathrm{~d}, 2 \mathrm{H}, J=$ 7.3); ${ }^{13} \mathrm{C}$ NMR (100 MHz) $\delta 22.9,43.5,59.2,70.6,125.5,127.5,127.7,128.6,128.9$, 132.1, 137.1, 145.1, 176.0. Anal. Calcd. for $\mathrm{C}_{19} \mathrm{H}_{23} \mathrm{NO}_{2} \mathrm{~S}: \mathrm{C}, 69.27 ; \mathrm{H}, 7.04 ; \mathrm{N}, 4.25$. Found: C, 69.40; H, 7.20; N, 4.37.

$\beta$-Hydroxy-N-Sulfinyl Imine 2f. General procedure A was followed with 203 $\mathrm{mg}(1.00 \mathrm{mmol})$ of $(R)-\mathbf{1 b}, 368 \mathrm{mg}(2.00 \mathrm{mmol})$ of $\mathrm{MgBr}_{2}$, and $95 \mathrm{mg}(1.10 \mathrm{mmol})$ of 
pivaldehyde. After the addition of the aldehyde, the reaction mixture was stirred for $3 \mathrm{~h}$ before the addition of $2 \mathrm{~N} \mathrm{AcOH} / \mathrm{THF}$. Diastereoselectivity was determined by HPLC analysis; 99:1 dr (98:2 hexanes/ $i$-PrOH, $1.0 \mathrm{~mL} / \mathrm{min}$ : major diastereomer, $\mathrm{t}_{\mathrm{R}}=4.9 \mathrm{~min}$; minor diastereomer, $\left.t_{R}=7.7 \mathrm{~min}\right)$. Column chromatography $(90: 10$ hexanes/EtOAc) afforded $266 \mathrm{mg}$ (92\%) of 2f: IR 1607, $3307 \mathrm{~cm}^{-1}$; ${ }^{1} \mathrm{H}$ NMR (500 MHz) $\delta 0.91(\mathrm{~s}, 9 \mathrm{H})$, 1.20 (s, 9H), 1.25 (s, 9H), 2.78 (dd, 1H, $J=12.0,3.3), 2.90$ (dd, 1H, $J=12.0,11.6$ ), 3.47 (ddd, $1 \mathrm{H}, J=11.6,10.0,3.3), 4.94(\mathrm{~d}, 1 \mathrm{H}, J=10.0) ;{ }^{13} \mathrm{C} \mathrm{NMR}(125 \mathrm{MHz}) \delta 22.7$, 25.7, 28.8, 35.7, 36.2, 43.7, 58.3, 75.0, 190.1. Anal. Calcd. for $\mathrm{C}_{15} \mathrm{H}_{31} \mathrm{NO}_{2} \mathrm{~S}: \mathrm{C}, 62.24 ; \mathrm{H}$, 10.79 ; N, 4.84. Found: C, 62.58; H, 11.03; N, 4.64.

$\beta$-Hydroxy- $\boldsymbol{N}$-Sulfinyl Imine 2g. General procedure A was followed with 203 $\mathrm{mg}(1.00 \mathrm{mmol})$ of $(R)-\mathbf{1 b}, 368 \mathrm{mg}(2.00 \mathrm{mmol})$ of $\mathrm{MgBr}_{2}$, and $79 \mathrm{mg}(1.10 \mathrm{mmol})$ of isobutyraldehyde. After the addition of the aldehyde, the reaction mixture was stirred for $3 \mathrm{~h}$ before the addition of $2 \mathrm{~N} \mathrm{AcOH} / \mathrm{THF}$. Diastereoselectivity was determined by HPLC analysis; 99:1 dr (98:2 hexanes $/ i-\mathrm{PrOH}, 1.0 \mathrm{~mL} / \mathrm{min}$ : major diastereomer, $\mathrm{t}_{\mathrm{R}}=$ $6.1 \mathrm{~min}$; minor diastereomer, $\left.t_{R}=7.6 \mathrm{~min}\right)$. Column chromatography $(90: 10$ hexanes/EtOAc) afforded $221 \mathrm{mg}(80 \%)$ of $\mathbf{2 g}$ : IR 1607, $3308 \mathrm{~cm}^{-1} ;{ }^{1} \mathrm{H}$ NMR (500 MHz) $\delta 0.91(\mathrm{~d}, 3 \mathrm{H}, J=6.7), 0.95(\mathrm{~d}, 3 \mathrm{H}, J=6.7), 1.19$ (s, 9H), 1.25 (s, 9H), 1.65 (dtt, $1 \mathrm{H}, J=5.8,6.7,6.7), 2.77(\mathrm{dd}, 1 \mathrm{H}, J=12.3,3.9), 2.89(\mathrm{dd}, 1 \mathrm{H}, J=12.3,11.1), 3.52$ (dddd, $1 \mathrm{H}, J=11.1,10.1,5.8,3.3), 4.78(\mathrm{~d}, 1 \mathrm{H}, J=10.1) ;{ }^{13} \mathrm{C}$ NMR (125 MHz) $\delta 17.9$, 18.7, 22.7, 28.5, 35.9, 38.6, 43.5, 58.2, 72.4, 189.9. Anal. Calcd. for $\mathrm{C}_{14} \mathrm{H}_{29} \mathrm{NO}_{2} \mathrm{~S}$ : C, $61.05 ; \mathrm{H}, 10.61 ; \mathrm{N}, 5.09$. Found: C, 61.23; H, 10.69; N, 4.91.

$\beta$-Hydroxy-N-Sulfinyl Imine $\mathbf{2 h}$. General procedure A was followed with 203 $\mathrm{mg}(1.00 \mathrm{mmol})$ of $(R)-\mathbf{1 b}, 368 \mathrm{mg}(2.00 \mathrm{mmol})$ of $\mathrm{MgBr}_{2}$, and $64 \mathrm{mg}(1.10 \mathrm{mmol})$ of propionaldehyde. After the addition of the aldehyde, the reaction mixture was stirred for $3 \mathrm{~h}$ before the addition of $2 \mathrm{~N}$ AcOH/THF. Diastereoselectivity was determined by HPLC analysis; 98:2 dr (97:3 hexanes $/ i-\mathrm{PrOH}, 1.0 \mathrm{~mL} / \mathrm{min}$ : major diastereomer, $\mathrm{t}_{\mathrm{R}}=$ $6.7 \mathrm{~min}$; minor diastereomer, $\left.t_{\mathrm{R}}=9.1 \mathrm{~min}\right)$. Column chromatography $(90: 10$ hexanes/EtOAc) afforded $220 \mathrm{mg}(84 \%)$ of $\mathbf{2 h}$ : IR 1604, $3361 \mathrm{~cm}^{-1} ;{ }^{1} \mathrm{H}$ NMR (500 MHz) $\delta 0.91(\mathrm{t}, 3 \mathrm{H}, J=7.4), 1.15(\mathrm{~s}, 9 \mathrm{H}), 1.21(\mathrm{~s}, 9 \mathrm{H}), 1.46(\mathrm{dq}, 2 \mathrm{H}, J=6.2,7.4), 2.75$ (dd, 1H, $J=12.4,4.7), 2.80(\mathrm{dd}, 1 \mathrm{H}, J=12.4,10.1), 3.65(\mathrm{~m}, 1 \mathrm{H}), 4.64(\mathrm{~d}, 1 \mathrm{H}, J=$ 10.2); ${ }^{13} \mathrm{C}$ NMR (125 MHz) $\delta 10.0,22.6,28.2,32.4,41.0,43.3,58.0,69.0,189.4$. Anal. Calcd. for $\mathrm{C}_{13} \mathrm{H}_{27} \mathrm{NO}_{2} \mathrm{~S}$ : C, 59.73; H, 10.41; N, 5.36. Found: C, 59.92; H, 10.79; N, 5.29 .

$\beta$-Hydroxy- $N$-Sulfinyl Imine 2i. General procedure A was followed with 203 $\mathrm{mg}(1.00 \mathrm{mmol})$ of $(R)-\mathbf{1 b}, 368 \mathrm{mg}(2.00 \mathrm{mmol})$ of $\mathrm{MgBr}_{2}$, and $117 \mathrm{mg}(1.10 \mathrm{mmol})$ of benzaldehyde. After the addition of the aldehyde, the reaction mixture was stirred for 1 
$\mathrm{h}$ before the addition of $2 \mathrm{~N}$ AcOH/THF. Diastereoselectivity was determined by HPLC analysis; 90:10 dr (97:3 hexanes $/ i-\mathrm{PrOH}, 1.0 \mathrm{~mL} / \mathrm{min}$ : major diastereomer, $\mathrm{t}_{\mathrm{R}}=5.3 \mathrm{~min}$; minor diastereomer, $\left.t_{R}=6.4 \mathrm{~min}\right)$. Column chromatography $(8: 1$ hexanes/EtOAc $)$ afforded $235 \mathrm{mg}$ (76\%) of 2i: IR 1602, $3345 \mathrm{~cm}^{-1} ;{ }^{1} \mathrm{H}$ NMR (500 MHz) $\delta 1.29(\mathrm{~s}, 9 \mathrm{H})$, 1.32 (s, 9H), $3.00(\mathrm{dd}, 1 \mathrm{H}, J=12.8,3.9), 3.18(\mathrm{dd}, 1 \mathrm{H}, J=12.8,11.3), 4.91(\mathrm{~m}, 1 \mathrm{H})$, $5.94(\mathrm{~d}, 1 \mathrm{H}, J=9.8), 7.21-7.43(\mathrm{~m}, 5 \mathrm{H}) ;{ }^{13} \mathrm{C} \mathrm{NMR}(125 \mathrm{MHz}) \delta 22.7,28.4,43.5,43.6$, 58.4, 70.0, 125.2, 127.1, 128.4, 145.4, 188.4. Anal. Calcd. for $\mathrm{C}_{17} \mathrm{H}_{27} \mathrm{NO}_{2} \mathrm{~S}: \mathrm{C}, 65.98 ; \mathrm{H}$, 8.79; N, 4.53. Found: C, 65.93; H, 9.12; N, 4.33.

$\boldsymbol{\beta}$-Hydroxy- $\boldsymbol{N}$-Sulfinyl Imine $\mathbf{2 j}$. General procedure A was followed with 189 $\mathrm{mg}(1.00 \mathrm{mmol})$ of $(R)-\mathbf{1 b}, 368 \mathrm{mg}(2.00 \mathrm{mmol})$ of $\mathrm{MgBr}_{2}$, and $95 \mathrm{mg}(1.10 \mathrm{mmol}) \mathrm{of}$ pivaldehyde. After the addition of the aldehyde, the reaction mixture was stirred for $3 \mathrm{~h}$ before the addition of $2 \mathrm{~N}$ AcOH/THF. Diastereoselectivity was determined by HPLC analysis; 99:1 dr (97:3 hexanes $/ i$-PrOH, $1.0 \mathrm{~mL} / \mathrm{min}$ : major diastereomer, $\mathrm{t}_{\mathrm{R}}=4.9 \mathrm{~min}$; minor diastereomer, $\left.t_{R}=6.4 \mathrm{~min}\right)$. Column chromatography $(8: 1$ hexanes/EtOAc) afforded $234 \mathrm{mg}(85 \%)$ of $2 \mathrm{e}$ : IR 1612, 1709, $3344 \mathrm{~cm}^{-1}$; ${ }^{1} \mathrm{H}$ NMR (500 MHz) $\delta 0.93$ (s, $9 \mathrm{H}), 1.14(\mathrm{~d}, 6 \mathrm{H}, J=6.8), 1.26(\mathrm{~s}, 9 \mathrm{H}), 2.54(\mathrm{dd}, 1 \mathrm{H}, J=11.8,3.0), 2.69(\mathrm{sep}, 1 \mathrm{H}, J=$ 6.8), $3.08(\mathrm{dd}, 1 \mathrm{H}, J=11.8,11.3), 3.39$ (ddd, $1 \mathrm{H}, J=11.3,8.9,3.0), 4.63(\mathrm{~d}, 1 \mathrm{H}, J=$ 8.9); ${ }^{13} \mathrm{C}$ NMR (125 MHz) $\delta 20.3,21.1,22.5,25.7,35.8,38.5,38.5,58.1,75.0,190.1$. Anal. Calcd. for $\mathrm{C}_{14} \mathrm{H}_{29} \mathrm{NO}_{2} \mathrm{~S}$ : C, 61.05; H, 10.61; N, 5.09. Found: C, 61.13; H, 10.77; $\mathrm{N}, 5.04$.

$\boldsymbol{\beta}$-Hydroxy- $\boldsymbol{N}$-Sulfinyl Imine 2k. General procedure A was followed with 189 $\mathrm{mg}(1.00 \mathrm{mmol})$ of $(R)-1 \mathrm{c}, 368 \mathrm{mg}(2.00 \mathrm{mmol})$ of $\mathrm{MgBr}_{2}$, and $79 \mathrm{mg}(1.10 \mathrm{mmol})$ of isobutyraldehyde. After the addition of the aldehyde, the reaction mixture was stirred for $3 \mathrm{~h}$ before the addition of $2 \mathrm{~N} \mathrm{AcOH} / \mathrm{THF}$. Diastereoselectivity was determined by HPLC analysis; 97:3 dr (97:3 hexanes $/ i-\mathrm{PrOH}, 1.0 \mathrm{~mL} / \mathrm{min}$ : major diastereomer, $\mathrm{t}_{\mathrm{R}}=$ $6.4 \mathrm{~min}$; minor diastereomer, $\left.t_{\mathrm{R}}=9.4 \mathrm{~min}\right)$. Column chromatography (6:1 hexanes/EtOAc) afforded $212 \mathrm{mg}(81 \%)$ of $2 \mathbf{k}$ : IR 1622, $3343 \mathrm{~cm}^{-1} ;{ }^{1} \mathrm{H}$ NMR (500 MHz) $\delta 0.94(\mathrm{~d}, 3 \mathrm{H}, J=6.8), 0.96(\mathrm{~d}, 3 \mathrm{H}, J=6.8), 1.15(\mathrm{~d}, 3 \mathrm{H}, J=6.8), 1.15(\mathrm{~d}, 3 \mathrm{H}, J$ $=6.8), 1.27(\mathrm{~s}, 9 \mathrm{H}), 1.69(\mathrm{dtt}, 1 \mathrm{H}, J=5.3,6.8,6.8), 2.52(\mathrm{dd}, 1 \mathrm{H}, J=11.8,3.3), 2.69$ (tt, $1 \mathrm{H}, J=6.8,6.8), 3.09(\mathrm{dd}, 1 \mathrm{H}, J=11.8,11.2), 3.52(\mathrm{dddd}, 1 \mathrm{H}, J=11.2,9.0,5.3,3.3)$, $4.54(\mathrm{~d}, 1 \mathrm{H}, J=9.0) ;{ }^{13} \mathrm{C}$ NMR $(125 \mathrm{MHz}) \delta 17.5,18.7,20.2,20.9,22.5,35.4,38.8$, 41.0, 58.1, 72.1, 189.7. Anal. Calcd. for $\mathrm{C}_{13} \mathrm{H}_{27} \mathrm{NO}_{2} \mathrm{~S}$ : C, 59.73; H, 10.41; N, 5.36. Found: C, 59.61; H, 10.66; N, 5.56.

$\beta$-Hydroxy- $\boldsymbol{N}$-Sulfinyl Imine 2l. General procedure A was followed with 189 $\mathrm{mg}(1.00 \mathrm{mmol})$ of $(R)-\mathbf{1 c}, 368 \mathrm{mg}(2.00 \mathrm{mmol})$ of $\mathrm{MgBr}_{2}$, and $64 \mathrm{mg}(1.10 \mathrm{mmol})$ of propionaldehyde. After the addition of the aldehyde, the reaction mixture was stirred for 
$3 \mathrm{~h}$ before the addition of $2 \mathrm{~N} \mathrm{AcOH} / \mathrm{THF}$. Diastereoselectivity was determined by HPLC analysis; 91:9 dr (97:3 hexanes $/ i-\mathrm{PrOH}, 1.0 \mathrm{~mL} / \mathrm{min}$ : major diastereomer, $\mathrm{t}_{\mathrm{R}}=$ $9.5 \mathrm{~min}$; minor diastereomer, $\left.t_{R}=15.1 \mathrm{~min}\right)$. Column chromatography (6:1 hexanes/EtOAc) afforded $181 \mathrm{mg}(73 \%)$ of 2l: IR 1622, 1707, $3356 \mathrm{~cm}^{-1} ;{ }^{1} \mathrm{H}$ NMR (500 MHz) $\delta 0.84(\mathrm{t}, 3 \mathrm{H}, J=7.4), 1.01(\mathrm{~d}, 6 \mathrm{H}, J=6.7), 1.13(\mathrm{~s}, 9 \mathrm{H}), 1.38-1.44(\mathrm{~m}, 2 \mathrm{H}), 2.48$ (dd, 1H, $J=12.0,3.4$ ), 2.56 (sep, 1H, $J=6.7$ ), 2.90 (dd, 1H, $J=12.0,10.7$ ), 3.57 (br s, $1 \mathrm{H}), 4.36(\mathrm{~d}, 1 \mathrm{H}, J=6.4) ;{ }^{13} \mathrm{C} \mathrm{NMR}(125 \mathrm{MHz}) \delta 9.6,19.8,20.5,22.2,38.7,43.1,57.6$, 68.5, 72.1, 189.1; HRMS (FAB) calcd for $[\mathrm{MH}]^{+}\left(\mathrm{C}_{12} \mathrm{H}_{26} \mathrm{NO}_{2} \mathrm{~S}\right) \mathrm{m} / z$ 248.1684. Found 248.1687 .

$\boldsymbol{\beta}$-Hydroxy- $\boldsymbol{N}$-Sulfinyl Imine $\mathbf{2 m}$. General procedure A was followed with 189 $\mathrm{mg}(1.00 \mathrm{mmol})$ of $(R)-\mathbf{1 c}, 368 \mathrm{mg}(2.00 \mathrm{mmol})$ of $\mathrm{MgBr}_{2}$, and $117 \mathrm{mg}(1.10 \mathrm{mmol})$ of benzaldehyde. After the addition of the aldehyde, the reaction mixture was stirred for 1 $\mathrm{h}$ before the addition of $2 \mathrm{~N} \mathrm{AcOH} / \mathrm{THF}$. Diastereoselectivity was determined by HPLC analysis; 77:23 dr (97:3 hexanes $/ i-\mathrm{PrOH}, 1.0 \mathrm{~mL} / \mathrm{min}$ : major diastereomer, $\mathrm{t}_{\mathrm{R}}=7.1 \mathrm{~min}$; minor diastereomer, $\left.t_{\mathrm{R}}=8.1 \mathrm{~min}\right)$. Column chromatography $(6: 1$ hexanes/EtOAc) afforded $220 \mathrm{mg}$ (75\%) of $\mathbf{2 m}$ : IR 1621, 1708, $3322 \mathrm{~cm}^{-1}$; ${ }^{1} \mathrm{H}$ NMR (500 MHz) $\delta 1.19$ $(\mathrm{d}, 3 \mathrm{H}, J=6.9), 1.21(\mathrm{~d}, 3 \mathrm{H}, J=6.6), 1.32(\mathrm{~s}, 9 \mathrm{H}), 2.79-2.88(\mathrm{~m}, 2 \mathrm{H}), 3.34(\mathrm{dd}, 1 \mathrm{H}, J=$ 12.0, 11.5), 4.86 (dd, $1 \mathrm{H}, J=12.0,3.2), 5.58$ (br s, $1 \mathrm{H}), 7.23-7.44(\mathrm{~m}, 5 \mathrm{H}) ;{ }^{13} \mathrm{C} \mathrm{NMR}$ (125 MHz) $\delta 20.2,20.9,22.5,39.0,46.3,58.2,69.8,125.4,127.3,128.4,145.1,188.3$. Anal. Calcd. for $\mathrm{C}_{16} \mathrm{H}_{25} \mathrm{NO}_{2} \mathrm{~S}: \mathrm{C}, 65.05 ; \mathrm{H}, 8.53 ; \mathrm{N}, 4.74$. Found: $\mathrm{C}, 65.02 ; \mathrm{H}, 8.54 ; \mathrm{N}$, 4.67 .

$\boldsymbol{\beta}$-Hydroxy- $\boldsymbol{N}$-Sulfinyl Imine 2n. General procedure A was followed with 175 $\mathrm{mg}(1.00 \mathrm{mmol})$ of $(R)-\mathbf{1 d}, 368 \mathrm{mg}(2.00 \mathrm{mmol})$ of $\mathrm{MgBr}_{2}$, and $95 \mathrm{mg}(1.10 \mathrm{mmol})$ of pivaldehyde. After the addition of the aldehyde, the reaction mixture was stirred for $3 \mathrm{~h}$ before the addition of $2 \mathrm{~N} \mathrm{AcOH} / \mathrm{THF}$. Diastereoselectivity was determined by HPLC analysis; 98:2 dr (97:3 hexanes $/ i-\mathrm{PrOH}, 1.0 \mathrm{~mL} / \mathrm{min}$ : major diastereomer, $\mathrm{t}_{\mathrm{R}}=6.1 \mathrm{~min}$; minor diastereomer, $\left.t_{R}=10.1 \mathrm{~min}\right)$. Column chromatography $(6: 1$ hexanes/EtOAc) afforded $182 \mathrm{mg}(70 \%)$ of $2 \mathrm{n}$ : IR 1579, 1708, 3349, $3240 \mathrm{~cm}^{-1} ;{ }^{1} \mathrm{H}$ NMR (500 MHz) $\delta$ 0.93 (s, 9H), 1.11 (t, 3H, $J=7.2), 1.27$ (s, 9H), 2.36 (dq, $1 \mathrm{H}, J=17.7,7.2), 2.44$ (dd, $1 \mathrm{H}$, $J=11.9,3.1), 2.66(\mathrm{dq}, 1 \mathrm{H}, J=17.7,7.2), 3.14$ (dd, $1 \mathrm{H}, J=11.9,11.4), 3.40$ (ddd, $1 \mathrm{H}$, $J=11.4,8.8,3.1), 4.60$ (d, $1 \mathrm{H}, J=8.8) ;{ }^{13} \mathrm{C} \mathrm{NMR}(125 \mathrm{MHz}) \delta 9.9,22.5,25.7,34.0$, 35.8, 39.7, 57.9, 75.4, 186.3. Anal. Calcd. for $\mathrm{C}_{13} \mathrm{H}_{27} \mathrm{NO}_{2} \mathrm{~S}: \mathrm{C}, 59.73 ; \mathrm{H}, 10.41 ; \mathrm{N}, 5.36$. Found: C, 59.43; H, 10.54; N, 5.51 .

$\beta$-Hydroxy- $N$-Sulfinyl Imine 2o. General procedure A was followed with 175 $\mathrm{mg}(1.00 \mathrm{mmol})$ of $(R)-\mathbf{1 d}, 368 \mathrm{mg}(2.00 \mathrm{mmol})$ of $\mathrm{MgBr}_{2}$, and $79 \mathrm{mg}(1.10 \mathrm{mmol})$ of isobutyraldehyde. After the addition of the aldehyde, the reaction mixture was stirred for 
$3 \mathrm{~h}$ before the addition of $2 \mathrm{~N} \mathrm{AcOH} / \mathrm{THF}$. Diastereoselectivity was determined by HPLC analysis; 92:8 dr (97:3 hexanes $/ i-\mathrm{PrOH}, 1.0 \mathrm{~mL} / \mathrm{min}$ : major diastereomer, $\mathrm{t}_{\mathrm{R}}=$ $8.8 \mathrm{~min}$; minor diastereomer, $\left.t_{R}=15.2 \mathrm{~min}\right)$. Column chromatography (4:1 hexanes/EtOAc) afforded $160 \mathrm{mg}(65 \%)$ of $2 \mathrm{o}$ : IR 1623, 1710, $3357 \mathrm{~cm}^{-1} ;{ }^{1} \mathrm{H}$ NMR $(500 \mathrm{MHz}) \delta 0.94(\mathrm{~d}, 3 \mathrm{H}, J=6.8), 0.95(\mathrm{~d}, 3 \mathrm{H}, J=6.8), 1.06(\mathrm{t}, 3 \mathrm{H}, J=7.3), 1.27$ (s, 9H), 1.69 (dtt, $1 \mathrm{H}, J=5.4,6.8,6.8), 2.36$ (dq, $1 \mathrm{H}, J=17.7,7.2), 2.41$ (dd, $1 \mathrm{H}, J=11.9$, 3.2), 2.64 (dq, $1 \mathrm{H}, J=17.7,7.2$ ), 3.14 (dd, $1 \mathrm{H}, J=11.9,11.0), 3.52$ (dddd, $1 \mathrm{H}, J=11.0$, 9.0, 5.4, 3.2), 4.50 (d, $1 \mathrm{H}, J=9.0) ;{ }^{13} \mathrm{C}$ NMR (125 MHz) $\delta 9.9,17.5,18.6,22.5,34.1$, 35.3, 42.1, 57.9, 72.4, 185.9. Anal. Calcd. for $\mathrm{C}_{12} \mathrm{H}_{25} \mathrm{NO}_{2} \mathrm{~S}: \mathrm{C}, 58.26 ; \mathrm{H}, 10.19 ; \mathrm{N}$, 5.66. Found: C, 58.25; H, 10.46; N, 5.87.

$\boldsymbol{\beta}$-Hydroxy- $\boldsymbol{N}$-Sulfinyl Imine 2p. General procedure A was followed with 525 $\mathrm{mg}(1.00 \mathrm{mmol})$ of $(R)-1 \mathrm{~d}, 1.10 \mathrm{~g}(2.00 \mathrm{mmol})$ of $\mathrm{MgBr}_{2}$, and $192 \mathrm{mg}(1.10 \mathrm{mmol})$ of propionaldehyde. After the addition of the aldehyde, the reaction mixture was stirred for $3 \mathrm{~h}$ before the addition of $2 \mathrm{~N} \mathrm{AcOH} / \mathrm{THF}$. Diastereoselectivity was determined by HPLC analysis; 80:20 dr (97:3 hexanes $/ i-\mathrm{PrOH}, 1.0 \mathrm{~mL} / \mathrm{min}$ : major diastereomer, $\mathrm{t}_{\mathrm{R}}=$ $16.0 \mathrm{~min}$; minor diastereomer, $\left.t_{\mathrm{R}}=26.8 \mathrm{~min}\right)$. Column chromatography $(5: 1$ hexanes/EtOAc) afforded $386 \mathrm{mg}(55 \%)$ of 2p: IR 1623, 1708, $3346 \mathrm{~cm}^{-1} ;{ }^{1} \mathrm{H}$ NMR (500 MHz) $\delta 0.96(\mathrm{t}, 3 \mathrm{H}, J=7.4), 1.09(\mathrm{t}, 3 \mathrm{H}, J=7.2), 1.25(\mathrm{~s}, 9 \mathrm{H}), 1.49-1.55(\mathrm{~m}, 2 \mathrm{H})$, 2.35 (dq, $1 \mathrm{H}, J=17.7,7.2), 2.46$ (dd, $1 \mathrm{H}, J=12.1,3.5), 2.62$ (dq, $1 \mathrm{H}, J=17.7,7.2$ ), $3.08(\mathrm{dd}, 1 \mathrm{H}, J=12.1,10.8), 3.64-3.70(\mathrm{~m}, 1 \mathrm{H}), 4.40(\mathrm{~d}, 1 \mathrm{H}, J=9.2) ;{ }^{13} \mathrm{C}$ NMR $(125$ MHz) $\delta 9.5,9.6,22.1,31.8,34.1,44.3,57.4,68.8,185.5$; HRMS (FAB) calcd for $[\mathrm{MH}]^{+}\left(\mathrm{C}_{11} \mathrm{H}_{24} \mathrm{NO}_{2} \mathrm{~S}\right) \mathrm{m} / \mathrm{z} 234.1528$. Found 234.1529.

$\beta$-Hydroxy- $N$-Sulfinyl Imine 2q. General procedure A was followed with 175 $\mathrm{mg}(1.00 \mathrm{mmol})$ of $(R)-\mathbf{1 d}, 368 \mathrm{mg}(2.00 \mathrm{mmol})$ of $\mathrm{MgBr}_{2}$, and $117 \mathrm{mg}(1.10 \mathrm{mmol})$ of benzaldehyde. After the addition of the aldehyde, the reaction mixture was stirred for 1 $\mathrm{h}$ before the addition of $2 \mathrm{~N} \mathrm{AcOH} / \mathrm{THF}$. Diastereoselectivity was determined by HPLC analysis; 60:40 dr (97:3 hexanes $/ i$-PrOH, $1.0 \mathrm{~mL} / \mathrm{min}$ : major diastereomer, $\mathrm{t}_{\mathrm{R}}=10.8$ $\mathrm{min}$; minor diastereomer, $\left.\mathrm{t}_{\mathrm{R}}=12.3 \mathrm{~min}\right)$. Column chromatography $(5: 1$ hexanes/EtOAc $)$ afforded $141 \mathrm{mg}$ (50\%) of 2q: IR 1709, 3250, $3357 \mathrm{~cm}^{-1} ;{ }^{1} \mathrm{H}$ NMR (500 MHz) $\delta 1.17$ (t, $3 \mathrm{H}, J=7.2), 1.31$ (s, 9H), $2.46(\mathrm{dq}, 1 \mathrm{H}, J=17.7,7.1), 2.69(\mathrm{dd}, 1 \mathrm{H}, J=12.4,3.7), 2.78$ (dq, $1 \mathrm{H}, J=17.7,7.1), 3.39$ (dd, $1 \mathrm{H}, J=12.4,11.3$ ), 4.86 (ddd, $1 \mathrm{H}, J=11.3,8.9,3.7$ ), $5.52(\mathrm{~d}, 1 \mathrm{H}, J=8.9), 7.23-7.42(\mathrm{~m}, 5 \mathrm{H}) ;{ }^{13} \mathrm{C} \mathrm{NMR}(125 \mathrm{MHz}) \delta 9.81,22.5,34.2,47.5$, 58.0, 70.0, 125.4, 127.3, 128.5, 145.0, 184.6. Anal. Calcd. for $\mathrm{C}_{15} \mathrm{H}_{23} \mathrm{NO}_{2} \mathrm{~S}: \mathrm{C}, 64.02 ; \mathrm{H}$, 8.24; N, 4.98. Found: C, 63.67; H, 8.39; N, 4.66.

General Procedure B for the Hydrolysis of $\boldsymbol{\beta}$-Hydroxy Sulfinyl Imines 2 . To a $0.02 \mathrm{M}$ solution of $\beta$-hydroxy sulfinyl imine 2 (1.0 equiv) in $\mathrm{MeOH}$ was added 1.0 
$N$ aqueous $\mathrm{AcOH}$ (20 equiv) and the mixture was stirred for $20 \mathrm{~h}$ at $40{ }^{\circ} \mathrm{C}$. After the hydrolysis was determined to be complete by TLC, the solvent was reduced to half volume under reduced pressure. Brine was added to the mixture, which was then extracted with 2:8 $\mathrm{CH}_{2} \mathrm{Cl}_{2}$ /hexanes $(3 \mathrm{x})$. The combined organic portions were dried, filtered, and concentrated. The $\beta$-hydroxy ketones were purified by silica gel chromatography.

(S)-3-Hydroxy-4-methyl-1-phenyl-1-pentanone (3c). The general procedure B was followed with $133 \mathrm{mg}(0.450 \mathrm{mmol})$ of $2 \mathrm{c}$. The enantiomeric excess was determined by HPLC analysis (Chiralpak AS, 98:2 hexanes/EtOH, $1.0 \mathrm{~mL} / \mathrm{min}, 210 \mathrm{~nm}$ : major enantiomer, $t_{R}=6.8 \mathrm{~min}$; minor enantiomer, $t_{R}=8.3 \mathrm{~min}$ ). Column chromatography (70:30 hexanes/EtOAc) delivered $85.0 \mathrm{mg}\left(98 \%, 98 \%\right.$ ee) of 3c: $[\alpha]_{\mathrm{D}}{ }^{23}$ -67.7 (c 1.43, $\mathrm{CHCl}_{3}$ ) [lit. value. ${ }^{5}[\alpha]_{\mathrm{D}}{ }^{23}-35.7$ (c $0.9, \mathrm{CHCl}_{3}, 47 \%$ ee)]. IR 1676, $3467 \mathrm{~cm}^{-1}$; ${ }^{1} \mathrm{H}$ NMR (500 MHz) $\delta 0.99(\mathrm{~d}, 3 \mathrm{H}, J=6.8), 1.01(\mathrm{~d}, 3 \mathrm{H}, J=6.8), 1.77-1.83$ (m, 1H), $3.03(\mathrm{dd}, 1 \mathrm{H}, J=9.6,17.4), 3.17(\mathrm{dd}, 1 \mathrm{H}, J=1.3,17.4), 3.21$ (br s, 1H), 3.99 (m, 1H), 7.47 (t, 2H, $J=7.4), 7.58(\mathrm{t}, 1 \mathrm{H}, J=7.4), 7.96$ (d, 2H, $J=7.4) ;{ }^{13} \mathrm{C}$ NMR $(125$ MHz) $\delta 17.9,18.5,33.1,42.0,72.3,128.0,128.7,133.4,136.9,201.3 . \quad$ IR, ${ }^{1} \mathrm{H}$ NMR and ${ }^{13} \mathrm{C}$ NMR spectroscopic data and optical rotation are in good agreement with those reported in literature. ${ }^{4,5}$ HRMS (FAB) calcd for $[\mathrm{MH}]^{+}\left(\mathrm{C}_{12} \mathrm{H}_{17} \mathrm{O}_{2}\right) \mathrm{m} / z$ 193.1229. Found 193.1235.

(S)-3-Hydroxy-1,3-diphenyl-1-propanone (3e). General procedure B was followed with $100 \mathrm{mg}(0.300 \mathrm{mmol})$ of $2 \mathbf{e}$. The enantiomeric excess was determined by HPLC analysis (Chiralpak AS, 94:6 hexanes/EtOH, $1.0 \mathrm{~mL} / \mathrm{min}, 210 \mathrm{~nm}$ : major enantiomer, $t_{R}=10.2 \mathrm{~min}$; minor enantiomer, $t_{R}=11.9 \mathrm{~min}$ ). Column chromatography (70:30 hexanes/EtOAc) delivered $69.0 \mathrm{mg}\left(>99 \%, 97 \%\right.$ ee) of 3e: $[\alpha]_{\mathrm{D}}{ }^{23}-94.0$ (c 1.29, $\left.\mathrm{CHCl}_{3}\right)$ [lit. value. ${ }^{6}[\alpha]_{\mathrm{D}}{ }^{24}-37.2\left(\mathrm{c} 1.28, \mathrm{CHCl}_{3}, 49 \%\right.$ ee)]. IR $1667,3465 \mathrm{~cm}^{-1} ;{ }^{1} \mathrm{H}$ NMR (400 MHz) $\delta 3.38(\mathrm{~d}, 2 \mathrm{H}, J=6.1), 3.61$ (br s, $1 \mathrm{H}), 5.36(\mathrm{t}, 1 \mathrm{H}, J=6.1), 7.29-7.62$ $(\mathrm{m}, 8 \mathrm{H}), 7.96(\mathrm{dd}, 2 \mathrm{H}, J=8.4,1.3) ;{ }^{13} \mathrm{C} \mathrm{NMR}(100 \mathrm{MHz}) \delta 47.3,70.0,125.6,127.7$, 128.0, 128.2, 128.6, 133.7, 136.5, 142.8, 200.2. IR, ${ }^{1} \mathrm{H}$ NMR and ${ }^{13} \mathrm{C}$ NMR spectroscopic data and optical rotation are in good agreement with those reported in literature. $^{4,5,6}$ Anal. Calcd. for $\mathrm{C}_{15} \mathrm{H}_{14} \mathrm{O}_{2}$ : C, 79.62; H, 6.24. Found: C, 79.28; H, 6.57.

General Procedure $\mathrm{C}$ for the Reduction of $\boldsymbol{\beta}$-Hydroxy Sulfinyl Imines 2 with Catecholborane. To a $0.200 \mathrm{M}$ solution of $\beta$-hydroxy sulfinyl imine 2 (1.0 equiv) in THF was added catecholborane (5.0 equiv), and the mixture was stirred for 20 $\mathrm{h}$ at $-10{ }^{\circ} \mathrm{C}$. To this mixture was added $\mathrm{MeOH}$ and a saturated solution of sodium potassium tartrate. The resulting mixture was stirred for $1 \mathrm{~h}$, washed with brine, and the brine layer was extracted with EtOAc $(2 x)$. The combined organic portions were 
dried, filtered, and concentrated. syn-1,3-Amino alcohols were isolated by column chromatography.

$\mathrm{N}$-Sulfinyl-1,3-Amino Alcohol syn-5a. General procedure $\mathrm{C}$ was followed with $93.0 \mathrm{mg}(0.30 \mathrm{mmol})$ of $\mathbf{2 a}$ and $180 \mathrm{mg}(1.50 \mathrm{mmol})$ of catecholborane. Diastereoselectivity was determined by HPLC analysis; $96: 4 \mathrm{dr}$ (94:6 hexanes/ $i-\mathrm{PrOH}$, $1.0 \mathrm{~mL} / \mathrm{min}: \mathrm{t}_{\mathrm{R}}$ (major) $=13.0 \mathrm{~min}, \mathrm{t}_{\mathrm{R}}($ minor $\left.)=6.2 \mathrm{~min}\right)$. Column chromatography (50:50 hexanes/EtOAc) afforded $83.0 \mathrm{mg}(89 \%)$ of syn-5a: IR $3194,3290 \mathrm{~cm}^{-1} ;{ }^{1} \mathrm{H}$ NMR (500 MHz) $\delta 0.84(\mathrm{~s}, 9 \mathrm{H}), 1.18(\mathrm{~s}, 9 \mathrm{H}), 1.93$ (ddd, 1H, $J=14.1,8.7,1.7), 2.10$ (ddd, $1 \mathrm{H}, J=14.1,10.6,6.9), 3.09$ (dd, 1H, $J=10.6,1.7), 4.10$ (apparent br d, 1H), $4.59(\mathrm{~m}, 1 \mathrm{H}), 7.27-7.38(\mathrm{~m}, 5 \mathrm{H}) ;{ }^{13} \mathrm{C}$ NMR $(100 \mathrm{MHz}) \delta 22.6,25.5,35.0,37.9,55.8$, 58.2, 78.0, 127.5, 127.8, 128.7, 142.0. Anal. Calcd. for $\mathrm{C}_{17} \mathrm{H}_{29} \mathrm{NO}_{2} \mathrm{~S}: \mathrm{C}, 65.55 ; \mathrm{H}$, 9.38; N, 4.50. Found: C, 65.46; H, 9.57; N, 4.26.

$\mathrm{N}$-Sulfinyl-1,3-Amino Alcohol syn-5b. General procedure $\mathrm{C}$ was followed with $89.0 \mathrm{mg}(0.300 \mathrm{mmol})$ of $\mathbf{2 b}$ and $180 \mathrm{mg}(1.50 \mathrm{mmol})$ of catecholborane. Diastereoselectivity was determined by HPLC analysis; 96:4 dr (94:6 hexanes/ $i$-PrOH, $1.0 \mathrm{~mL} / \mathrm{min}: \mathrm{t}_{\mathrm{R}}$ (major) $=15.8 \mathrm{~min}, \mathrm{t}_{\mathrm{R}}($ minor $\left.)=8.4 \mathrm{~min}\right)$. Column chromatography (50:50 hexanes/EtOAc) afforded $78.0 \mathrm{mg}(88 \%)$ of $\mathbf{s y n - 5 b}:$ IR $3175,3244 \mathrm{~cm}^{-1} ;{ }^{1} \mathrm{H}$ NMR (400 MHz) $\delta 0.85(\mathrm{~d}, 6 \mathrm{H}, J=6.8), 1.18(\mathrm{~s}, 9 \mathrm{H}), 1.56-1.64(\mathrm{~m}, 1 \mathrm{H}), 1.84(\mathrm{ddd}, 1 \mathrm{H}$, $J=2.0,7.8,14.0), 2.01(\mathrm{ddd}, 1 \mathrm{H}, J=6.8,10.3,14.0), 2.67$ (br s, 1H), $3.23(\mathrm{ddd}, 1 \mathrm{H}, J=$ 2.0, 5.1, 10.3), 4.08 (br s, $1 \mathrm{H}), 4.61$ (br t, 1H), 7.25-7.38 (m, 5H); ${ }^{13} \mathrm{C} \mathrm{NMR} \mathrm{(125} \mathrm{MHz)}$ $\delta 17.3,18.5,22.6,34.3,40.6,55.8,57.8,74.6,127.4,127.8,128.6,142.2$. Anal. Calcd. for $\mathrm{C}_{16} \mathrm{H}_{27} \mathrm{O}_{2} \mathrm{~S}$ : C, 64.60; H, 9.15; N, 4.71. Found: C, 64.51; H, 9.04; N, 4.38.

$\mathrm{N}$-Sulfinyl-1,3-Amino Alcohol syn-5c. General procedure $\mathrm{C}$ was followed with $93.0 \mathrm{mg}(0.300 \mathrm{mmol})$ of $2 \mathrm{c}$ and $180 \mathrm{mg}(1.50 \mathrm{mmol})$ of catecholborane. Diastereoselectivity was determined by HPLC analysis; 96:4 dr (97:3 hexanes/ $i-\mathrm{PrOH}$, $1.0 \mathrm{~mL} / \mathrm{min}: \mathrm{t}_{\mathrm{R}}$ (major) $=52.6 \mathrm{~min}, \mathrm{t}_{\mathrm{R}}($ minor $\left.)=20.0 \mathrm{~min}\right)$. Column chromatography (50:50 hexanes/EtOAc) afforded $78.0 \mathrm{mg}(84 \%)$ of syn-5c: IR $3273,3380 \mathrm{~cm}^{-1} ;{ }^{1} \mathrm{H}$ NMR (400 MHz) $\delta 0.83(\mathrm{~d}, 3 \mathrm{H}, J=6.7), 0.83(\mathrm{~d}, 3 \mathrm{H}, J=6.7), 1.19(\mathrm{~s}, 9 \mathrm{H}), 1.21-1.27$ (m, 1H), 1.41 (ddd, $1 \mathrm{H}, J=14.1,8.5,5.8), 1.71(\mathrm{~m}, 1 \mathrm{H}), 1.85$ (ddd, $1 \mathrm{H}, J=14.2,7.2$, 2.6), 2.15 (ddd, $1 \mathrm{H}, J=14.2,9.8,7.2$ ), 3.59 (dddd, $1 \mathrm{H}, J=9.8,8.5,4.7,2.6$ ), 4.03 (br s, $1 \mathrm{H}), 4.62(\mathrm{dd}, 1 \mathrm{H}, J=7.2,7.2), 7.27-7.38(\mathrm{~m}, 5 \mathrm{H}) ;{ }^{13} \mathrm{C}$ NMR $(100 \mathrm{MHz}) \delta 22.2,22.6$, 23.1, 24.4, 44.3, 47.7, 55.9, 57.7, 68.4, 127.3, 127.9, 128.7, 142.1. Anal. Calcd. for $\mathrm{C}_{17} \mathrm{H}_{29} \mathrm{NO}_{2} \mathrm{~S}$ : C, 65.55; H, 9.38; N, 4.50. Found: C, 65.17; H, 9.45; N, 4.43.

$\boldsymbol{N}$-Sulfinyl-1,3-Amino Alcohol syn-5d. General procedure $\mathrm{C}$ was followed with $84.0 \mathrm{mg}(0.300 \mathrm{mmol})$ of $\mathbf{2 d}$ and $180 \mathrm{mg}(1.50 \mathrm{mmol})$ of catecholborane. Diastereoselectivity was determined by HPLC analysis; 95:5 dr (94:6 hexanes/i-PrOH, 
$1.0 \mathrm{~mL} / \mathrm{min}: \mathrm{t}_{\mathrm{R}}($ major $)=19.6 \mathrm{~min}, \mathrm{t}_{\mathrm{R}}($ minor $\left.)=11.2 \mathrm{~min}\right)$. Column chromatography (70:30 hexanes/EtOAc) afforded $79.0 \mathrm{mg}(94 \%)$ of $\mathbf{s y n - 5 d : ~ I R ~ 3 2 9 8 , ~} 3391 \mathrm{~cm}^{-1} ;{ }^{1} \mathrm{H}$ $\operatorname{NMR}(500 \mathrm{MHz}) \delta 0.88(\mathrm{t}, 3 \mathrm{H}, J=7.4), 1.19(\mathrm{~s}, 9 \mathrm{H}), 1.46(\mathrm{dq}, 2 \mathrm{H}, J=7.4,6.2), 1.85$ (ddd, $1 \mathrm{H}, J=14.1,7.8,2.4), 2.18$ (ddd, $1 \mathrm{H}, J=14.1,10.1,6.6), 3.42$ (ddt, $1 \mathrm{H}, J=10.1$, 6.2, 2.4), $4.60(\mathrm{dd}, 1 \mathrm{H}, J=7.8,6.6), 7.28-7.37(\mathrm{~m}, 5 \mathrm{H}) ;{ }^{13} \mathrm{C} \mathrm{NMR}(100 \mathrm{MHz}) \delta 9.7$, 22.6, 31.1, 43.2, 55.9, 57.3, 71.4, 127.4, 128.0, 128.8, 142.0. Anal. Calcd. for $\mathrm{C}_{15} \mathrm{H}_{25} \mathrm{NO}_{2} \mathrm{~S}$ : C, 63.56; H, 8.89; N, 4.94. Found: C, 63.59; H, 8.87; N, 4.67.

$\mathrm{N}$-Sulfinyl-1,3-Amino Alcohol syn-5e. General procedure $\mathrm{C}$ was followed with $99.0 \mathrm{mg}(0.300 \mathrm{mmol})$ of $2 \mathrm{e}$ and $180 \mathrm{mg}(1.50 \mathrm{mmol})$ of catecholborane. Diastereoselectivity was determined by HPLC analysis; 96:4 dr (94:6 hexanes/i-PrOH, $1.0 \mathrm{~mL} / \mathrm{min}: \mathrm{t}_{\mathrm{R}}($ major $)=18.8 \mathrm{~min}, \mathrm{t}_{\mathrm{R}}($ minor $\left.)=10.2 \mathrm{~min}\right)$. Column chromatography (50:50 hexanes/EtOAc) afforded $83.0 \mathrm{mg}(84 \%)$ of syn-5e: IR $3178,3242 \mathrm{~cm}^{-1} ;{ }^{1} \mathrm{H}$ NMR (400 MHz) $\delta 1.20(\mathrm{~s}, 9 \mathrm{H}), 2.04(\mathrm{ddd}, 1 \mathrm{H}, J=14.2,7.2,3.2), 2.54$ (ddd, 1H, $J=$ $14.2,10.1,6.6), 4.17(\mathrm{~d}, 1 \mathrm{H}, J=4.1), 4.52$ (dd, $1 \mathrm{H}, J=10.1,3.2), 4.69(\mathrm{~m}, 1 \mathrm{H})$, 7.22-7.40 (m, 10H); ${ }^{13} \mathrm{C}$ NMR (100 MHz) $\delta 22.6,45.9,56.0,57.4,72.1,125.6,127.4$, 127.5, 128.0, 128.5, 128.7, 141.8, 144.6. Anal. Calcd. for $\mathrm{C}_{19} \mathrm{H}_{25} \mathrm{NO}_{2} \mathrm{~S}: \mathrm{C}, 68.85 ; \mathrm{H}$, 7.60; N, 4.23. Found: C, 68.51; H, 7.88; N, 4.15.

$\boldsymbol{N}$-Sulfinyl-1,3-Amino Alcohol syn-5f. General procedure B was followed with $58 \mathrm{mg}(0.200 \mathrm{mmol})$ of $\mathbf{2 f}$ and $120 \mathrm{mg}(1.00 \mathrm{mmol})$ of catecholborane. Diastereoselectivity was determined by HPLC analysis; 95:5 dr (98:2 hexanes/EtOH, $1.0 \mathrm{~mL} / \mathrm{min}$ : major diastereomer, $\mathrm{t}_{\mathrm{R}}=13.4 \mathrm{~min}$; minor diastereomer, $\mathrm{t}_{\mathrm{R}}=8.7 \mathrm{~min}$ ). Column chromatography (7:3 hexanes/EtOAc) afforded $46 \mathrm{mg}$ (79\%) of syn-5f: IR 3260, $3294 \mathrm{~cm}^{-1}$; ${ }^{1} \mathrm{H}$ NMR (500 MHz) $\delta 0.90(\mathrm{~s}, 9 \mathrm{H}), 0.96(\mathrm{~s}, 9 \mathrm{H}), 1.24(\mathrm{~s}, 9 \mathrm{H}), 1.85$ (ddd, $1 \mathrm{H}, J=15.3,10.0,8.8 \mathrm{~Hz}), 1.91$ (ddd, $1 \mathrm{H}, J=15.3,3.0,2.6 \mathrm{~Hz}), 3.01$ (ddd, $1 \mathrm{H}, J$ $=8.8,6.4,2.6 \mathrm{~Hz}), 3.22(\mathrm{dd}, 1 \mathrm{H}, J=10.0,3.0 \mathrm{~Hz}), 4.02(\mathrm{~d}, 1 \mathrm{H}, J=6.4 \mathrm{~Hz}) ;{ }^{13} \mathrm{C} \mathrm{NMR}$ $(125 \mathrm{MHz}) \delta 22.8,25.7,27.3,31.2,35.5,36.0,56.5,65.4,81.7$. Anal. Calcd. for $\mathrm{C}_{15} \mathrm{H}_{33} \mathrm{NO}_{2} \mathrm{~S}: \mathrm{C}, 61.81 ; \mathrm{H}, 11.41 ; \mathrm{N}, 4.81$. Found: C, 61.95; H, 11.54; N, 4.42.

$\boldsymbol{N}$-Sulfinyl-1,3-Amino Alcohol syn-5g. General procedure B was followed with $55 \mathrm{mg}(0.200 \mathrm{mmol})$ of $\mathbf{2 g}$ and $120 \mathrm{mg}(1.00 \mathrm{mmol})$ of catecholborane. Diastereoselectivity was determined by HPLC analysis; 96:4 dr (97:3 hexanes/ $i$-PrOH, $1.0 \mathrm{~mL} / \mathrm{min}$ : major diastereomer, $\mathrm{t}_{\mathrm{R}}=29.4 \mathrm{~min}$; minor diastereomer, $\mathrm{t}_{\mathrm{R}}=12.0 \mathrm{~min}$ ). Column chromatography (5:5 hexanes/EtOAc) afforded $50 \mathrm{mg}(91 \%)$ of $\boldsymbol{s y n - 5 g : ~ I R ~}$ $3211,3384 \mathrm{~cm}^{-1}$; ${ }^{1} \mathrm{H}$ NMR (500 MHz) $\delta 0.90(\mathrm{~d}, 3 \mathrm{H}, J=6.8 \mathrm{~Hz}), 0.93(\mathrm{~d}, 3 \mathrm{H}, J=6.8$ $\mathrm{Hz}), 0.96(\mathrm{~s}, 9 \mathrm{H}), 1.23(\mathrm{~s}, 9 \mathrm{H}), 1.68(\mathrm{dtt}, 1 \mathrm{H}, J=4.5,6.8,6.8 \mathrm{~Hz}), 1.80(\mathrm{ddd}, 1 \mathrm{H}, J=$ 15.0, 4.6, $2.6 \mathrm{~Hz}$ ), 1.86 (ddd, $1 \mathrm{H}, J=15.0,9.2,9.0 \mathrm{~Hz}$ ), 3.00 (ddd, $1 \mathrm{H}, J=9.2,6.5,2.6$ $\mathrm{Hz}), 3.33$ (d, 1H, $J=7.2 \mathrm{~Hz}), 3.43$ (br s, $1 \mathrm{H}), 3.88$ (d, $1 \mathrm{H}, J=6.5 \mathrm{~Hz}) ;{ }^{13} \mathrm{C}$ NMR $(125$ 
MHz) $\delta$ 16.6, 18.9, 22.8, 27.2, 34.0, 34.5, 35.6, 56.5, 64.6, 77.3. Anal. Calcd. for $\mathrm{C}_{14} \mathrm{H}_{31} \mathrm{NO}_{2} \mathrm{~S}$ : C, 60.60; H, 11.26; N, 5.05. Found: C, 60.48; H, 11.49; N, 4.82.

$\mathrm{N}$-Sulfinyl-1,3-Amino Alcohol syn-5h. General procedure B was followed with $52 \mathrm{mg}(0.200 \mathrm{mmol})$ of $\mathbf{2 h}$ and $120 \mathrm{mg}(1.00 \mathrm{mmol})$ of catecholborane. Diastereoselectivity was determined by HPLC analysis; 95:5 dr (97:3 hexanes/ $i-\mathrm{PrOH}$, $1.0 \mathrm{~mL} / \mathrm{min}$ : major diastereomer, $t_{R}=48.2 \mathrm{~min}$; minor diastereomer, $t_{R}=19.3 \mathrm{~min}$ ). Column chromatography (4:6 hexanes/EtOAc) afforded $42 \mathrm{mg}(50 \%)$ of $\boldsymbol{s y n}-\mathbf{5 h}$ : IR $3208,3359 \mathrm{~cm}^{-1}$; ${ }^{1} \mathrm{H}$ NMR (500 MHz) $\delta 0.95$ (dd, $\left.1 \mathrm{H}, J=7.5,7.5 \mathrm{~Hz}\right), 0.95(\mathrm{~s}, 9 \mathrm{H})$, 1.23 (s, 9H), 1.44 (ddq, $1 \mathrm{H}, J=15.0,6.5,7.5 \mathrm{~Hz}), 1.56$ (ddq, $1 \mathrm{H}, J=15.0,4.4,7.5 \mathrm{~Hz}$ ), 1.78 (ddd, $1 \mathrm{H}, J=14.8,5.7,2.8 \mathrm{~Hz}$ ), 1.83 (ddd, $1 \mathrm{H}, J=14.8,9.4,7.9 \mathrm{~Hz}$ ), 2.99 (ddd, $1 \mathrm{H}, J=9.4,6.5,2.8 \mathrm{~Hz}), 3.05(\mathrm{~d}, 1 \mathrm{H}, J=7.1 \mathrm{~Hz}), 3.60(\mathrm{~m}, 1 \mathrm{H}), 3.76(\mathrm{~d}, 1 \mathrm{H}, J=6.5$ $\mathrm{Hz}) ;{ }^{13} \mathrm{C}$ NMR $(125 \mathrm{MHz}) \delta 9.7,22.8,27.1,30.7,35.4,37.7,56.4,64.1,73.4$. Anal. Calcd. for $\mathrm{C}_{13} \mathrm{H}_{29} \mathrm{NO}_{2} \mathrm{~S}: \mathrm{C}, 59.27 ; \mathrm{H}, 11.10 ; \mathrm{N}, 5.32$. Found: $\mathrm{C}, 59.04 ; \mathrm{H}, 11.17 ; \mathrm{N}$, 5.06 .

N-Sulfinyl-1,3-Amino Alcohol syn-5i. General procedure B was followed with $62 \mathrm{mg}(0.200 \mathrm{mmol})$ of $\mathbf{2 i}$ and $120 \mathrm{mg}(1.00 \mathrm{mmol})$ of catecholborane. Diastereoselectivity was determined by HPLC analysis; 98:2 dr (97:3 hexanes/ $i-\mathrm{PrOH}$, $1.0 \mathrm{~mL} / \mathrm{min}$ : major diastereomer, $\mathrm{t}_{\mathrm{R}}=45.3 \mathrm{~min}$; minor diastereomer, $\mathrm{t}_{\mathrm{R}}=9.5 \mathrm{~min}$ ). Column chromatography (5:5 hexanes/EtOAc) afforded $43 \mathrm{mg}(69 \%)$ of syn-5i: IR $3154,3227 \mathrm{~cm}^{-1}$; ${ }^{1} \mathrm{H}$ NMR $(500 \mathrm{MHz}) \delta 0.88(\mathrm{~s}, 9 \mathrm{H}), 1.29(\mathrm{~s}, 9 \mathrm{H}), 2.05$ (ddd, 1H, $J=$ 14.7, 6.7, $1.9 \mathrm{~Hz}$ ), 2.13 (ddd, $1 \mathrm{H}, J=14.7,9.4,7.8 \mathrm{~Hz}$ ), 2.92 (ddd, $1 \mathrm{H}, J=9.4,6.8,1.9$ $\mathrm{Hz}), 3.80(\mathrm{~d}, 1 \mathrm{H}, J=6.8 \mathrm{~Hz}), 4.76(\mathrm{dd}, 1 \mathrm{H}, J=7.8,6.7 \mathrm{~Hz}), 7.24-7.39(\mathrm{~m}, 5 \mathrm{H}) ;{ }^{13} \mathrm{C}$ NMR (125 MHz) $\delta 22.9,26.9,35.3,40.4,56.6,63.4,74.7,126.1,127.6,128.5,144.5$; HRMS (FAB) calcd for $[\mathrm{MH}]^{+}\left(\mathrm{C}_{17} \mathrm{H}_{30} \mathrm{NO}_{2} \mathrm{~S}\right) \mathrm{m} / \mathrm{z} 312.1997$. Found 312.1998.

$\boldsymbol{N}$-Sulfinyl-1,3-Amino Alcohol syn-5j. General procedure B was followed with $55 \mathrm{mg}(0.200 \mathrm{mmol})$ of $\mathbf{2} \mathbf{j}$ and $120 \mathrm{mg}(1.00 \mathrm{mmol})$ of catecholborane. Diastereoselectivity was determined by HPLC analysis; 95:5 dr (97:3 hexanes/EtOH, $1.0 \mathrm{~mL} / \mathrm{min}$ : major diastereomer, $\mathrm{t}_{\mathrm{R}}=20.2 \mathrm{~min}$; minor diastereomer, $\mathrm{t}_{\mathrm{R}}=8.6 \mathrm{~min}$ ). Column chromatography (5:5 hexanes/EtOAc) afforded $51 \mathrm{mg}(93 \%)$ of $\boldsymbol{s y n}-\mathbf{5 j}$ : IR $3277,3411 \mathrm{~cm}^{-1}$; ${ }^{1} \mathrm{H}$ NMR $(500 \mathrm{MHz}) \delta 0.87(\mathrm{~s}, 9 \mathrm{H}), 0.92(\mathrm{~d}, 3 \mathrm{H}, J=6.8 \mathrm{~Hz}), 0.95(\mathrm{~d}$, $3 \mathrm{H}, J=6.8 \mathrm{~Hz}), 1.21(\mathrm{~s}, 9 \mathrm{H}), 1.53-1.60(\mathrm{~m}, 1 \mathrm{H}), 1.75-1.79(\mathrm{~m}, 1 \mathrm{H}), 2.07(\mathrm{dtt}, 1 \mathrm{H}, J=$ 6.1, 6.8, $6.8 \mathrm{~Hz}$ ), 3.09-3.14 (m, 1H), 3.27 (apparent br d, 1H, $J=9.0 \mathrm{~Hz}), 3.94$ (d, 1H, $J$ $=6.3 \mathrm{~Hz}) ;{ }^{13} \mathrm{C}$ NMR $(125 \mathrm{MHz}) \delta 18.7,19.1,22.6,25.6,32.0,32.9,35.3,56.1,62.9$, 80.3. Anal. Calcd. for $\mathrm{C}_{14} \mathrm{H}_{31} \mathrm{NO}_{2} \mathrm{~S}: \mathrm{C}, 60.60 ; \mathrm{H}, 11.26 ; \mathrm{N}, 5.05$. Found: C, 60.72; H, $11.41 ; \mathrm{N}, 4.80$.

N-Sulfinyl-1,3-Amino Alcohol syn-5k. General procedure B was followed 
with $52 \mathrm{mg}(0.200 \mathrm{mmol})$ of $\mathbf{2 k}$ and $120 \mathrm{mg}(1.00 \mathrm{mmol})$ of catecholborane. Diastereoselectivity was determined by HPLC analysis; 96:4 dr (97:3 hexanes/EtOH, $1.0 \mathrm{~mL} / \mathrm{min}$ : major diastereomer, $t_{R}=38.8 \mathrm{~min}$; minor diastereomer, $t_{R}=12.9 \mathrm{~min}$ ). Column chromatography (5:5 hexanes/EtOAc) afforded $43 \mathrm{mg}(82 \%)$ of $\boldsymbol{s y n - 5 k}$ : IR $3209,3315 \mathrm{~cm}^{-1}$; ${ }^{1} \mathrm{H}$ NMR $(500 \mathrm{MHz}) \delta 0.88(\mathrm{~d}, 3 \mathrm{H}, J=6.8 \mathrm{~Hz}), 0.91(\mathrm{~d}, 3 \mathrm{H}, J=6.8$ $\mathrm{Hz}), 0.93(\mathrm{~d}, 3 \mathrm{H}, J=6.9 \mathrm{~Hz}), 0.94(\mathrm{~d}, 3 \mathrm{H}, J=6.9 \mathrm{~Hz}), 1.21(\mathrm{~s}, 9 \mathrm{H}), 1.58-1.69(\mathrm{~m}, 3 \mathrm{H})$, 2.08 (dtt, $1 \mathrm{H}, J=5.9,6.8,6.8 \mathrm{~Hz}), 3.12-3.16(\mathrm{~m}, 1 \mathrm{H}), 3.46-3.50(\mathrm{~m}, 1 \mathrm{H}), 3.86$ (br s, $1 \mathrm{H}) ;{ }^{13} \mathrm{C}$ NMR (125 MHz) $\delta 16.7,18.3,18.7,19.1,22.6,32.1,34.0,35.3,56.1,62.3$, 76.5. Anal. Calcd. for $\mathrm{C}_{13} \mathrm{H}_{29} \mathrm{NO}_{2} \mathrm{~S}: \mathrm{C}, 59.27 ; \mathrm{H}, 11.10 ; \mathrm{N}, 5.32$. Found: C, 58.97; H, $11.22 ; \mathrm{N}, 4.93$.

N-Sulfinyl-1,3-Amino Alcohol syn-5l. General procedure B was followed with $49 \mathrm{mg}(0.200 \mathrm{mmol})$ of $\mathbf{2 l}$ and $120 \mathrm{mg}(1.00 \mathrm{mmol})$ of catecholborane. Diastereoselectivity was determined by HPLC analysis; 95:5 dr (97:3 hexanes/EtOH, $1.0 \mathrm{~mL} / \mathrm{min}$ : major diastereomer, $\mathrm{t}_{\mathrm{R}}=49.3 \mathrm{~min}$; minor diastereomer, $\mathrm{t}_{\mathrm{R}}=16.4 \mathrm{~min}$ ). Column chromatography (3:7 hexanes/EtOAc) afforded $40 \mathrm{mg}(82 \%)$ of syn-5l: IR $3178,3317 \mathrm{~cm}^{-1}$; ${ }^{1} \mathrm{H}$ NMR (500 MHz) $\delta$ 0.91-0.94 (m, 9H), $1.21(\mathrm{~s}, 9 \mathrm{H}), 1.38-1.66(\mathrm{~m}$, $4 \mathrm{H}), 2.02-2.11(\mathrm{~m}, 1 \mathrm{H}), 3.02$ (br s, $1 \mathrm{H}), 3.13-3.18(\mathrm{~m}, 1 \mathrm{H}), 3.58-3.64(\mathrm{~m}, 1 \mathrm{H}), 3.75(\mathrm{~d}$, $1 \mathrm{H}, J=7.0 \mathrm{~Hz}) ;{ }^{13} \mathrm{C}$ NMR $(125 \mathrm{MHz}) \delta 9.6,17.8,19.1,22.6,30.8,32.2,38.0,56.1$, 62.0, 72.9. Anal. Calcd. for $\mathrm{C}_{12} \mathrm{H}_{27} \mathrm{NO}_{2} \mathrm{~S}: \mathrm{C}, 57.79 ; \mathrm{H}, 10.91 ; \mathrm{N}, 5.62$. Found: $\mathrm{C}$, $57.91 ; \mathrm{H}, 11.03 ; \mathrm{N}, 5.26$.

$\mathrm{N}$-Sulfinyl-1,3-Amino Alcohol syn-5m. General procedure B was followed with $89 \mathrm{mg}(0.300 \mathrm{mmol})$ of $\mathbf{2 m}$ and $120 \mathrm{mg}(1.00 \mathrm{mmol})$ of catecholborane. Diastereoselectivity was determined by HPLC analysis; 90:10 dr (94:6 hexanes/EtOH, $1.0 \mathrm{~mL} / \mathrm{min}$ : major diastereomer, $\mathrm{t}_{\mathrm{R}}=23.8 \mathrm{~min}$; minor diastereomer, $\mathrm{t}_{\mathrm{R}}=6.6 \mathrm{~min}$ ). Column chromatography (5:5 hexanes/EtOAc) afforded $73 \mathrm{mg}(81 \%)$ of syn-5m: IR $3206,3276 \mathrm{~cm}^{-1}$; ${ }^{1} \mathrm{H}$ NMR $(500 \mathrm{MHz}) \delta 0.87(\mathrm{~d}, 3 \mathrm{H}, J=6.8 \mathrm{~Hz}), 0.92(\mathrm{~d}, 3 \mathrm{H}, J=6.8$ $\mathrm{Hz}), 1.25$ (s, 9H), 1.86-2.10 (m, 3H), 3.11-3.15 (m, 1H), 3.55 (br s, 1H), 3.75 (d, 1H, $J=$ $7.4 \mathrm{~Hz}), 4.76-4.80(\mathrm{~m}, 1 \mathrm{H}), 7.25-7.38(\mathrm{~m}, 5 \mathrm{H}) ;{ }^{13} \mathrm{C} \mathrm{NMR}(125 \mathrm{MHz}) \delta 17.7,19.0,22.7$, 32.5, 40.8, 56.2, 61.3, 74.1, 125.9, 127.6, 128.5, 144.6. Anal. Calcd. for $\mathrm{C}_{16} \mathrm{H}_{27} \mathrm{NO}_{2} \mathrm{~S}: \mathrm{C}$, 64.60; H, 9.15; N, 4.71. Found: C, 64.22; H, 9.20; N, 4.60.

$\mathrm{N}$-Sulfinyl-1,3-Amino Alcohol syn-5n. General procedure B was followed with $52 \mathrm{mg}(0.200 \mathrm{mmol})$ of $2 \mathrm{n}$ and $120 \mathrm{mg}(1.00 \mathrm{mmol})$ of catecholborane. Diastereoselectivity was determined by HPLC analysis; $92: 8 \mathrm{dr}$ (97:3 hexanes/EtOH, $1.0 \mathrm{~mL} / \mathrm{min}$ : major diastereomer, $\mathrm{t}_{\mathrm{R}}=25.0 \mathrm{~min}$; minor diastereomer, $\mathrm{t}_{\mathrm{R}}=13.5 \mathrm{~min}$ ). Column chromatography (5:5 hexanes/EtOAc) afforded $43 \mathrm{mg}(82 \%)$ of $\boldsymbol{s y n - 5 n}$ : IR 3256, $3392 \mathrm{~cm}^{-1}$; ${ }^{1} \mathrm{H}$ NMR (500 MHz) $\delta 0.87(\mathrm{~s}, 9 \mathrm{H}), 0.95$ (t, 3H, $\left.J=7.3 \mathrm{~Hz}\right), 1.22$ (s, 
9H), 1.59-1.82 (m, 4H), 1.93 (br s, $1 \mathrm{H}), 3.24-3.33(\mathrm{~m}, 2 \mathrm{H}), 4.10(\mathrm{~d}, 1 \mathrm{H}, J=3.8 \mathrm{~Hz}),{ }^{13} \mathrm{C}$ NMR (125 MHz) $\delta 10.6,22.6,25.6,28.2,35.3,36.7,55.8,59.1,80.3$. Anal. Calcd. for $\mathrm{C}_{13} \mathrm{H}_{29} \mathrm{NO}_{2} \mathrm{~S}$ : C, 59.27; H, 11.10; N, 5.32. Found: C, 58.89; H, 11.40; N, 4.94.

$\mathrm{N}$-Sulfinyl-1,3-Amino Alcohol syn-5o. General procedure B was followed with $49 \mathrm{mg}(0.200 \mathrm{mmol})$ of $2 \mathrm{o}$ and $120 \mathrm{mg}(1.00 \mathrm{mmol})$ of catecholborane. Diastereoselectivity was determined by HPLC analysis; 97:3 dr (97:3 hexanes/EtOH, $1.0 \mathrm{~mL} / \mathrm{min}$ : major diastereomer, $\mathrm{t}_{\mathrm{R}}=43.4 \mathrm{~min}$; minor diastereomer, $\mathrm{t}_{\mathrm{R}}=18.2 \mathrm{~min}$ ). Column chromatography (5:5 hexanes/EtOAc) afforded $45 \mathrm{mg}(91 \%)$ of syn-5o: IR $3216,3334 \mathrm{~cm}^{-1}$; ${ }^{1} \mathrm{H}$ NMR (500 MHz) $\delta 0.87(\mathrm{~d}, 3 \mathrm{H}, J=6.8 \mathrm{~Hz}), 0.89$ (d, 3H, $J=6.9$ Hz), 0.93 (t, 3H, $J=7.4$ Hz), 1.19 (s, 9H), 1.57-1.65 (m, 3H), 1.70-1.80 (m, 2H), 3.18 (br s, $1 \mathrm{H}), 3.21-3.27(\mathrm{~m}, 1 \mathrm{H}), 3.47-3.50(\mathrm{~m}, 1 \mathrm{H}), 3.98(\mathrm{~d}, 1 \mathrm{H}, J=4.1 \mathrm{~Hz}) ;{ }^{13} \mathrm{C} \mathrm{NMR}$ (125 MHz) $\delta 10.5,16.9,18.6,22.5,28.3,34.4,39.3,55.7,58.6,76.6$. Anal. Calcd. for $\mathrm{C}_{12} \mathrm{H}_{27} \mathrm{NO}_{2} \mathrm{~S}$ : C, 57.79; H, 10.91; N, 5.62. Found: C, 57.58; H, 10.84; N, 5.95.

$\mathrm{N}$-Sulfinyl-1,3-Amino Alcohol syn-5p. General procedure B was followed with $70 \mathrm{mg}(3.00 \mathrm{mmol})$ of $\mathbf{2 p}$ and $180 \mathrm{mg}(15.0 \mathrm{mmol})$ of catecholborane. Diastereoselectivity was determined by HPLC analysis; 94:6 dr (94:6 hexanes/EtOH, $1.0 \mathrm{~mL} / \mathrm{min}$ : major diastereomer, $\mathrm{t}_{\mathrm{R}}=19.8 \mathrm{~min}$; minor diastereomer, $\mathrm{t}_{\mathrm{R}}=9.4 \mathrm{~min}$ ). Column chromatography (2:8 hexanes/EtOAc) afforded $53 \mathrm{mg}(76 \%)$ of syn-5p: IR $3207,3327 \mathrm{~cm}^{-1}$; ${ }^{1} \mathrm{H}$ NMR (500 MHz) $\delta 0.92(\mathrm{t}, 3 \mathrm{H}, J=7.3 \mathrm{~Hz}), 0.94(\mathrm{t}, 3 \mathrm{H}, J=7.3$ Hz), 1.21 (s, 9H), 1.40-1.80 (m, 6H), 2.03 (br s, 1H), 3.24-3.30 (m, 1H), 3.64 (br s, 1H), $3.88(\mathrm{~d}, 1 \mathrm{H}, J=5.5 \mathrm{~Hz}) ;{ }^{13} \mathrm{C}$ NMR $(125 \mathrm{MHz}) \delta 9.6,10.4,22.6,28.6,31.2,42.0,55.8$, 58.4, 73.1. Anal. Calcd. for $\mathrm{C}_{11} \mathrm{H}_{25} \mathrm{NO}_{2} \mathrm{~S}: \mathrm{C}, 56.13 ; \mathrm{H}, 10.71 ; \mathrm{N}, 5.95$. Found: $\mathrm{C}$, $56.25 ; \mathrm{H}, 10.75 ; \mathrm{N}, 5.65$.

N-Sulfinyl-1,3-Amino Alcohol syn-5q. General procedure B was followed with $56 \mathrm{mg}(0.200 \mathrm{mmol})$ of $\mathbf{2 q}$ and $120 \mathrm{mg}(1.00 \mathrm{mmol})$ of catecholborane. Diastereoselectivity was determined by HPLC analysis; 76:24 dr (94:6 hexanes/EtOH, $1.0 \mathrm{~mL} / \mathrm{min}$ : major diastereomer, $\mathrm{t}_{\mathrm{R}}=24.3 \mathrm{~min}$; minor diastereomer, $\mathrm{t}_{\mathrm{R}}=8.3 \mathrm{~min}$ ). Column chromatography (7:3 hexanes/EtOAc) afforded $39 \mathrm{mg}(69 \%)$ of syn-5q: IR $3187,3284 \mathrm{~cm}^{-1}$; ${ }^{1} \mathrm{H}$ NMR (500 MHz) $\delta 0.93$ (t, 3H, $\left.J=7.4 \mathrm{~Hz}\right), 1.24$ (s, 9H), 1.59-1.67 $(\mathrm{m}, 1 \mathrm{H}), 1.72-1.81(\mathrm{~m}, 1 \mathrm{H}), 1.86(\mathrm{ddd}, 1 \mathrm{H}, J=14.7,3.9,3.9 \mathrm{~Hz}), 2.09$ (ddd, 1H, $J=$ 14.7, 9.6, $9.6 \mathrm{~Hz}), 3.27-3.34$ (m, 1H), 3.76 (br s, 1H), 3.85 (d, 1H, $J=5.9 \mathrm{~Hz}), 4.80$ (dd, $1 \mathrm{H}, J=9.6,3.9 \mathrm{~Hz}), 7.23-7.37(\mathrm{~m}, 5 \mathrm{H}) ;{ }^{13} \mathrm{C} \mathrm{NMR}(125 \mathrm{MHz}) \delta 10.3,22.6,28.7,44.7$, 55.9, 57.9, 74.0, 125.6, 127.4, 128.5, 144.9. Anal. Calcd. for $\mathrm{C}_{15} \mathrm{H}_{25} \mathrm{NO}_{2} \mathrm{~S}: \mathrm{C}, 63.56 ; \mathrm{H}$, 8.89; N, 4.94. Found: C, 63.55; H, 8.99; N, 4.55.

General Procedure D for the Reduction of $\boldsymbol{\beta}$-Hydroxy Sulfinyl Imines 2 with LiBHEt $_{3}$. To a $-78{ }^{\circ} \mathrm{C}$ solution of $\beta$-hydroxy $N$-sulfinyl imine 2 (1.00 equiv) in 
THF $(0.200 \mathrm{M})$ was added $\mathrm{LiBHEt}_{3}(2.50$ equiv, $1.00 \mathrm{M}$ in THF) and the resulting solution was stirred for $3 \mathrm{~h}$. To this solution was added $1.00 \mathrm{~mL}$ of a saturated aqueous solution of $\mathrm{NH}_{4} \mathrm{Cl}$. The resulting mixture was gradually warmed to $\mathrm{rt}$ with stirring, washed with brine, and the brine layer was extracted with EtOAc (2x). The combined organic portions were dried, filtered, and concentrated. anti-1,3-Amino alcohols were isolated by column chromatography.

$\mathbf{N}$-Sulfinyl-1,3-Amino Alcohol anti-5a. General procedure D was followed with $155 \mathrm{mg}(0.500 \mathrm{mmol})$ of $\mathbf{2 a}$ and $1.25 \mathrm{~mL}$ of $\mathrm{LiBHEt}_{3}(1.25 \mathrm{mmol}, 1.00 \mathrm{M}$ in THF). Diastereoselectivity was determined by HPLC analysis, $>99: 1 \mathrm{dr}(94: 6$ hexanes $/ i-\mathrm{PrOH}$, $1.0 \mathrm{~mL} / \mathrm{min}: \mathrm{t}_{\mathrm{R}}($ major $)=6.1 \mathrm{~min}, \mathrm{t}_{\mathrm{R}}($ minor $\left.)=12.9 \mathrm{~min}\right)$; LCMS analysis, $>99: 1 \mathrm{dr}, \mathrm{MH}^{+}$ Calcd: 312.5 . Found: $312.1(\mathrm{C} 8,15 \mathrm{~cm})$ 70-90\% MeOH / $\mathrm{H}_{2} \mathrm{O} / 0.10 \% \mathrm{TFA}, \mathrm{t}_{\mathrm{R}}$ (major) $=18.0 \mathrm{~min}, \mathrm{t}_{\mathrm{R}}($ minor $\left.)=17.5 \mathrm{~min}\right)$. Column chromatography $(70: 30$ hexanes $/$ EtOAc $)$ afforded $113 \mathrm{mg}(73.0 \%)$ of anti-5a: $[\alpha]_{\mathrm{D}}^{23}=-90.4\left(\mathrm{c}=1.0, \mathrm{CHCl}_{3}\right)$; IR $3226 \mathrm{~cm}^{-1} ;{ }^{1} \mathrm{H}$ NMR (500 MHz) $\delta 0.85(\mathrm{~s}, 9 \mathrm{H}), 1.24(\mathrm{~s}, 9 \mathrm{H}), 1.39-1.52(\mathrm{~m}, 1 \mathrm{H}), 1.75-1.96(\mathrm{~m}, 2 \mathrm{H})$, 3.34-3.37 (m, 1H), $4.16(\mathrm{~d}, 1 \mathrm{H}, J=6.6), 4.73-4.78(\mathrm{~m}, 1 \mathrm{H}), 7.23-7.35(\mathrm{~m}, 5 \mathrm{H}) ;{ }^{13} \mathrm{C}$ NMR (125 MHz) $\delta 22.7,25.0,34.8,39.6,55.7,57.6,74.9,126.4,126.9,128.3,143.2$. Anal. Calcd. for $\mathrm{C}_{17} \mathrm{H}_{29} \mathrm{NO}_{2} \mathrm{~S}: \mathrm{C}, 65.55 ; \mathrm{H}, 9.38 ; \mathrm{N}, 4.50$. Found: C, 65.65; H, 9.31; N, 4.35 .

N-Sulfinyl-1,3-Amino Alcohol anti-5b. General procedure D was followed with $148 \mathrm{mg}(0.500 \mathrm{mmol})$ of $\mathbf{2} \mathbf{b}$ and $1.25 \mathrm{~mL}$ of $\operatorname{LiBHEt}_{3}(1.25 \mathrm{mmol}, 1.00 \mathrm{M}$ in THF). Diastereoselectivity was determined by HPLC analysis, $>99: 1 \mathrm{dr}(94: 6$ hexanes/ $i-\mathrm{PrOH}$, $1.0 \mathrm{~mL} / \mathrm{min}: \mathrm{t}_{\mathrm{R}}$ (major) $=8.31 \mathrm{~min}, \mathrm{t}_{\mathrm{R}}($ minor $\left.)=14.6 \mathrm{~min}\right)$; LCMS analysis, $>99: 1 \mathrm{dr}$, $\mathrm{MH}^{+}$Calcd: 297.5. Found: 297.3 (C8, $\left.15 \mathrm{~cm}\right)$ 70-90\% $\mathrm{MeOH} / \mathrm{H}_{2} \mathrm{O} /$ 0.10\% TFA, $t_{R}($ major $)=17.0 \mathrm{~min}, t_{R}($ minor $\left.)=16.4 \mathrm{~min}\right)$. Column chromatography $(70: 30$ hexanes/EtOAc) afforded $126 \mathrm{mg}(85.0 \%)$ of anti-5b: $[\alpha]_{\mathrm{D}}{ }^{23}=-92.7\left(\mathrm{c}=1.0, \mathrm{CHCl}_{3}\right)$; IR $3220 \mathrm{~cm}^{-1}$; ${ }^{1} \mathrm{H}$ NMR (500 MHz) $\delta 0.88(\mathrm{~d}, 3 \mathrm{H}, J=6.8), 0.93(\mathrm{~d}, 3 \mathrm{H}, J=6.7), 1.23(\mathrm{~s}$, 9H), 1.68-1.75 (m, 1H), 1.85-1.90 (m, 1H), 1.98-2.03 (m, 1H), 3.46-3.50 (m, 1H), 4.29 (br s, 1H), 4.74 (br d, 1H), 7.23-7.45 (m, 5H); ${ }^{13} \mathrm{C}$ NMR (125 MHz) $\delta 18.0,18.8,22.7$, 33.6, 42.2, 55.7, 57.0, 72.9, 126.5, 127.1, 128.4, 143.3. Anal. Calcd for $\mathrm{C}_{16} \mathrm{H}_{27} \mathrm{NO}_{2} \mathrm{~S}: \mathrm{C}$, 64.60; H, 9.15; N, 4.71. Found: C, 64.52; H, 9.03; N, 4.64.

$\boldsymbol{N}$-Sulfinyl-1,3-Amino Alcohol anti-5c. General procedure D was followed with $155 \mathrm{mg}(0.500 \mathrm{mmol})$ of $2 \mathrm{c}$ and $1.25 \mathrm{~mL}$ of $\mathrm{LiBHEt}_{3}(1.25 \mathrm{mmol}, 1.00 \mathrm{M}$ in THF). Diastereoselectivity was determined by HPLC analysis, $>99: 1 \mathrm{dr}(94: 6$ hexanes $/ i-\mathrm{PrOH}$, $1.0 \mathrm{~mL} / \mathrm{min}: \mathrm{t}_{\mathrm{R}}($ major $)=7.65 \mathrm{~min}, \mathrm{t}_{\mathrm{R}}($ minor $\left.)=15.8 \mathrm{~min}\right)$; LCMS analysis, $>99: 1 \mathrm{dr}$, $\mathrm{MH}^{+}$Calcd: 312.5. Found: $312.1(\mathrm{C} 8,15 \mathrm{~cm})$ 70-90\% $\mathrm{MeOH} / \mathrm{H}_{2} \mathrm{O} / 0.10 \%$ TFA, $\mathrm{t}_{\mathrm{R}}($ major $)=18.2 \mathrm{~min}, \mathrm{t}_{\mathrm{R}}($ minor $\left.)=17.8 \mathrm{~min}\right)$. Column chromatography $(70: 30$ 
hexanes/EtOAc) afforded $129 \mathrm{mg}(83.0 \%)$ of anti-5c: $[\alpha]_{\mathrm{D}}{ }^{23}=-99.8\left(\mathrm{c}=1.0, \mathrm{CHCl}_{3}\right)$; IR $3224 \mathrm{~cm}^{-1}$; ${ }^{1} \mathrm{H}$ NMR (500 MHz) $\delta 0.88(\mathrm{~m}, 6 \mathrm{H}), 1.24(\mathrm{~s}, 9 \mathrm{H}), 1.38-1.46(\mathrm{~m}, 1 \mathrm{H})$, 1.51-1.61 (m, 2H), 1.73-1.76 (m, 1H), 1.89-1.94 (m, 2H), 3.85-3.88 (m, 1H), $4.07(\mathrm{t}, 1 \mathrm{H}$, $J=6.7), 4.73(\mathrm{dd}, 1 \mathrm{H}, J=8.6,4.4), 7.23-7.36(\mathrm{~m}, 5 \mathrm{H}) ;{ }^{13} \mathrm{C}$ NMR $(125 \mathrm{MHz}) \delta 22.2$, 22.6, 23.1, 24.5, 45.2, 45.9, 55.5, 56.5, 66.3, 126.6, 127.0, 128.3, 143.1. Anal. Calcd. for $\mathrm{C}_{17} \mathrm{H}_{29} \mathrm{NO}_{2} \mathrm{~S}: \mathrm{C}, 65.55 ; \mathrm{H}, 9.38 ; \mathrm{N}, 4.50$. Found: C, 65.35; H, 9.29; N, 4.43.

$\boldsymbol{N}$-Sulfinyl-1,3-Amino Alcohol anti-5d. General procedure D was followed with $141 \mathrm{mg}(0.500 \mathrm{mmol})$ of $\mathbf{2 d}$ and $1.25 \mathrm{~mL}$ of $\operatorname{LiBHEt}_{3}(1.25 \mathrm{mmol}, 1.00 \mathrm{M}$ in THF). Diastereoselectivity was determined by HPLC analysis, $>99: 1 \mathrm{dr}(94: 6$ hexanes $/ i-\mathrm{PrOH}$, $1.0 \mathrm{~mL} / \mathrm{min}: \mathrm{t}_{\mathrm{R}}($ major $)=12.1 \mathrm{~min}, \mathrm{t}_{\mathrm{R}}($ minor $\left.)=19.6 \mathrm{~min}\right)$; LCMS analysis, $>99: 1 \mathrm{dr}$, $\mathrm{MH}^{+}$Calcd: 284.4. Found: $284.1(\mathrm{C} 8,15 \mathrm{~cm})$ 70-90\% $\mathrm{MeOH} / \mathrm{H}_{2} \mathrm{O} / 0.10 \%$ TFA, $\mathrm{t}_{\mathrm{R}}$ (major) $=15.4 \mathrm{~min}, \mathrm{t}_{\mathrm{R}}($ minor $\left.)=14.8 \mathrm{~min}\right)$. Column chromatography $(70: 30$ hexanes/EtOAc) afforded $97.8 \mathrm{mg}(67.0 \%)$ of anti-5d: $[\alpha]_{\mathrm{D}}{ }^{23}=-134.0\left(\mathrm{c}=1.0, \mathrm{CHCl}_{3}\right)$; IR $3225 \mathrm{~cm}^{-1}$; ${ }^{1} \mathrm{H}$ NMR (500 MHz) $\delta 0.93(\mathrm{t}, 3 \mathrm{H}, J=7.4), 1.22(\mathrm{~s}, 9 \mathrm{H}), 1.50-1.62(\mathrm{~m}$, 2H), 1.88-1.98 (m, 2H), 3.68-3.69 (m, 1H), 4.54 (br s, 1H), 4.72 (br d, 1H), 7.23-7.45 $(\mathrm{m}, 5 \mathrm{H}) ;{ }^{13} \mathrm{C} \mathrm{NMR}(125 \mathrm{MHz}) \delta 10.1,22.7,29.8,44.4,55.6,56.6,69.8,126.6,127.1$, 128.4, 143.2. Anal. Calcd. for $\mathrm{C}_{15} \mathrm{H}_{25} \mathrm{NO}_{2} \mathrm{~S}$ : C, 63.56; H, 8.89; N, 4.94. Found: $\mathrm{C}$, $63.45 ; \mathrm{H}, 8.93 ; \mathrm{N}, 4.76$.

$\mathrm{N}$-Sulfinyl-1,3-Amino Alcohol anti-5e. General procedure D was followed with $165 \mathrm{mg}(0.500 \mathrm{mmol})$ of $2 \mathrm{e}$ and $1.25 \mathrm{~mL}$ of $\mathrm{LiBHEt}_{3}(1.25 \mathrm{mmol}, 1.00 \mathrm{M}$ in THF). Diastereoselectivity was determined by HPLC analysis, $>99: 1 \mathrm{dr}(94: 6$ hexanes $/ i-\mathrm{PrOH}$, $1.0 \mathrm{~mL} / \mathrm{min}: \mathrm{t}_{\mathrm{R}}$ (major) $=10.6 \mathrm{~min}, \mathrm{t}_{\mathrm{R}}($ minor $\left.)=18.8 \mathrm{~min}\right)$; LCMS analysis, $>99: 1 \mathrm{dr}$, $\mathrm{MH}^{+}$Calcd: 312.5. Found: $312.1(\mathrm{C} 8,15 \mathrm{~cm})$ 70-90\% $\mathrm{MeOH} / \mathrm{H}_{2} \mathrm{O} / 0.10 \%$ TFA, $\mathrm{t}_{\mathrm{R}}($ major $)=17.0 \mathrm{~min}, \mathrm{t}_{\mathrm{R}}($ minor $\left.)=16.4 \mathrm{~min}\right)$. Column chromatography $(70: 30$ hexanes/EtOAc) afforded $151 \mathrm{mg}(91.0 \%)$ of anti-5e: $[\alpha]_{\mathrm{D}}^{23}=-121.6\left(\mathrm{c}=1.0, \mathrm{CHCl}_{3}\right)$; IR 1615, $3228 \mathrm{~cm}^{-1}$; ${ }^{1} \mathrm{H}$ NMR (500 MHz) $\delta 1.22(\mathrm{~s}, 9 \mathrm{H}), 2.15-2.31(\mathrm{~m}, 2 \mathrm{H}), 4.07(\mathrm{t}, 1 \mathrm{H}$, $J=6.7), 4.50$ (br s, $1 \mathrm{H}), 4.65-4.67(\mathrm{~m}, 1 \mathrm{H}), 4.86$ (dd, $1 \mathrm{H}, J=8.3,4.1), 7.20-7.37$ (m, $10 \mathrm{H}) ;{ }^{13} \mathrm{C}$ NMR (125 MHz) $\delta 22.6,46.7,55.6,56.7,71.0,125.8,126.7,127.5,128.4$, 128.4, 128.4, 142.6, 143.7. Anal. Calcd. for $\mathrm{C}_{16} \mathrm{H}_{27} \mathrm{NO}_{2} \mathrm{~S}: \mathrm{C}, 68.85 ; \mathrm{H}, 7.60 ; \mathrm{N}, 4.23$. Found: C, 68.72; H, 7.73; N, 4.19.

N-Sulfinyl-1,3-Amino Alcohol anti-5f. General procedure C was followed with $58 \mathrm{mg}(0.200 \mathrm{mmol})$ of $\mathbf{2} \mathbf{f}$, and $0.50 \mathrm{~mL}$ of $\mathrm{LiBHEt}_{3}(0.50 \mathrm{mmol}, 1.00 \mathrm{M}$ in THF). Diastereoselectivity was determined by HPLC analysis; >99:1 dr (98:2 hexanes/EtOH, $1.0 \mathrm{~mL} / \mathrm{min}$ : major diastereomer, $t_{R}=8.5 \mathrm{~min}$; minor diastereomer, $t_{R}=12.5 \mathrm{~min}$ ). Column chromatography (8:2 hexanes/EtOAc) afforded $48 \mathrm{mg}(83 \%)$ of anti-5f: IR 3340, $3378 \mathrm{~cm}^{-1}$; ${ }^{1} \mathrm{H}$ NMR (500 MHz) $\delta 0.88$ (s, 9H), 0.89 (s, 9H), 1.27 (s, 9H), 1.29 
(ddd, $1 \mathrm{H}, J=14.3,11.4,2.6 \mathrm{~Hz}), 1.71(\mathrm{ddd}, 1 \mathrm{H}, J=14.3,11.6,3.3 \mathrm{~Hz}), 2.98(\mathrm{~d}, 1 \mathrm{H}, J=$ $7.4 \mathrm{~Hz}), 3.20(\mathrm{ddd}, 1 \mathrm{H}, J=11.4,7.4,3.3 \mathrm{~Hz}), 3.32(\mathrm{ddd}, 1 \mathrm{H}, J=11.6,6.0,2.6 \mathrm{~Hz}), 3.98$ $(\mathrm{d}, 1 \mathrm{H}, J=6.0 \mathrm{~Hz}) ;{ }^{13} \mathrm{C}$ NMR $(125 \mathrm{MHz}) \delta 23.0,26.1,26.6,31.4,34.8,35.5,56.3,61.6$, 73.3. Anal. Calcd. for $\mathrm{C}_{15} \mathrm{H}_{33} \mathrm{NO}_{2} \mathrm{~S}$ : C, 61.81; H, 11.41; N, 4.81. Found: $\mathrm{C}, 61.59 ; \mathrm{H}$, $11.68 ; \mathrm{N}, 4.69$.

N-Sulfinyl-1,3-Amino Alcohol anti-5g. General procedure C was followed with $55 \mathrm{mg}(0.200 \mathrm{mmol})$ of $\mathbf{2 g}$, and $0.50 \mathrm{~mL}$ of $\operatorname{LiBHEt}_{3}(0.50 \mathrm{mmol}, 1.00 \mathrm{M}$ in THF $)$. Diastereoselectivity was determined by HPLC analysis; $>99: 1 \mathrm{dr}(97: 3$ hexanes/i-PrOH, $1.0 \mathrm{~mL} / \mathrm{min}$ : major diastereomer, $\mathrm{t}_{\mathrm{R}}=9.7 \mathrm{~min}$; minor diastereomer, $\mathrm{t}_{\mathrm{R}}=23.0 \mathrm{~min}$ ). Column chromatography (7:3 hexanes/EtOAc) afforded $53 \mathrm{mg}(96 \%)$ of anti-5g: IR $3340,3378 \mathrm{~cm}^{-1}$; ${ }^{1} \mathrm{H}$ NMR $(500 \mathrm{MHz}) \delta 0.86(\mathrm{~s}, 9 \mathrm{H}), 0.88(\mathrm{~d}, 3 \mathrm{H}, J=6.7 \mathrm{~Hz}), 0.95(\mathrm{~d}$, $3 \mathrm{H}, J=6.7 \mathrm{~Hz}), 1.25$ (s, 9H), $1.34(\mathrm{ddd}, 1 \mathrm{H}, J=14.4,10.5,2.8 \mathrm{~Hz}), 1.65-1.71(\mathrm{~m}, 1 \mathrm{H})$, 1.73 (ddd, $1 \mathrm{H}, J=14.4,10.5,3.1 \mathrm{~Hz}$ ), 3.10 (d, $1 \mathrm{H}, J=7.0 \mathrm{~Hz}$ ), 3.19 (ddd, $1 \mathrm{H}, J=11.5$, 7.1, 3.1 Hz), 3.34-3.40 (m, 1H), $4.13(\mathrm{~d}, 1 \mathrm{H}, J=6.3 \mathrm{~Hz}) ;{ }^{13} \mathrm{C}$ NMR $(125 \mathrm{MHz}) \delta 18.6$, 19.1, 22.9, 26.4, 33.6, 34.4, 35.4, 56.3, 61.2, 71.5. Anal. Calcd. for $\mathrm{C}_{14} \mathrm{H}_{31} \mathrm{NO}_{2} \mathrm{~S}: \mathrm{C}$, 60.60; H, 11.26; N, 5.05. Found: C, 60.44; H, 11.20; N, 4.74.

$\mathrm{N}$-Sulfinyl-1,3-Amino Alcohol anti-5h. General procedure C was followed with $52 \mathrm{mg}(0.200 \mathrm{mmol})$ of $\mathbf{2} \mathbf{h}$, and $0.50 \mathrm{~mL}$ of $\mathrm{LiBHEt}_{3}(0.50 \mathrm{mmol}, 1.00 \mathrm{M}$ in THF). Diastereoselectivity was determined by HPLC analysis; $>99: 1 \mathrm{dr}(97: 3$ hexanes/ $i-\mathrm{PrOH}$, $1.0 \mathrm{~mL} / \mathrm{min}$ : major diastereomer, $t_{R}=15.1 \mathrm{~min}$; minor diastereomer, not observed). Column chromatography (6:4 hexanes/EtOAc) afforded $50 \mathrm{mg}(96 \%)$ of anti-5h: IR $3272,3319 \mathrm{~cm}^{-1}$; ${ }^{1} \mathrm{H}$ NMR (500 MHz) $\delta 0.86(\mathrm{~s}, 9 \mathrm{H}), 0.93(\mathrm{t}, 1 \mathrm{H}, J=7.4 \mathrm{~Hz}), 1.25$ (s, 9H), 1.37 (ddd, 1H, $J=14.4,11.4,2.8 \mathrm{~Hz}), 1.43$ (ddq, 1H, $J=13.6,4.9,7.4 \mathrm{~Hz}), 1.53$ (ddq, $1 \mathrm{H}, J=13.6,7.1,7.4 \mathrm{~Hz}), 1.67$ (ddd, 1H, $J=14.4,10.2,3.0 \mathrm{~Hz}$ ), 3.14 (d, 1H, $J=$ $6.9 \mathrm{~Hz}$ ), 3.19 (ddd, $1 \mathrm{H}, J=11.4,6.9,3.0 \mathrm{~Hz}$ ), 3.55-3.63 (m, 1H), 4.21 (br s, $1 \mathrm{H}) ;{ }^{13} \mathrm{C}$ NMR (125 MHz) $\delta 10.6,22.9,26.4,29.8,35.3,37.2,56.3,61.0,68.1$. Anal. Calcd. for $\mathrm{C}_{13} \mathrm{H}_{29} \mathrm{NO}_{2} \mathrm{~S}$ : C, 59.27; H, 11.10; N, 5.32. Found: C, 59.21; H, 11.31; N, 5.13.

$\mathbf{N}$-Sulfinyl-1,3-Amino Alcohol anti-5i. General procedure C was followed with $62 \mathrm{mg}(0.200 \mathrm{mmol})$ of $2 \mathbf{i}$, and $0.50 \mathrm{~mL}$ of $\mathrm{LiBHEt}_{3}(0.50 \mathrm{mmol}, 1.00 \mathrm{M}$ in THF $)$. Diastereoselectivity was determined by HPLC analysis; $>99: 1 \mathrm{dr}(98: 2$ hexanes $/ i-\mathrm{PrOH}$, $1.0 \mathrm{~mL} / \mathrm{min}$ : major diastereomer, $t_{R}=9.1 \mathrm{~min}$; minor diastereomer, $t_{R}=44.4 \mathrm{~min}$ ). Column chromatography (5:5 hexanes/EtOAc) afforded $59 \mathrm{mg}(95 \%)$ of anti-5i: IR $3215,3335,3347 \mathrm{~cm}^{-1}$; ${ }^{1} \mathrm{H}$ NMR (500 MHz) $\delta 0.89$ (s, 9H), 1.32 (s, 9H), 1.61 (ddd, 1H, $J=14.4,11.3,2.8 \mathrm{~Hz}), 2.04(\mathrm{ddd}, 1 \mathrm{H}, J=14.4,10.8,2.9 \mathrm{~Hz}), 3.24(\mathrm{~d}, 1 \mathrm{H}, J=7.5 \mathrm{~Hz})$, 3.32 (ddd, $1 \mathrm{H}, J=11.3,7.5,2.9 \mathrm{~Hz}), 4.67$ (br s, $1 \mathrm{H}), 7.22-7.40(\mathrm{~m}, 5 \mathrm{H}) ;{ }^{13} \mathrm{C}$ NMR $(125$ MHz) $\delta$ 23.0, 26.4, 35.4, 40.3, 56.4, 61.5, 68.8, 125.6, 126.8, 128.2, 144.7; HRMS 
(FAB) calcd for $[\mathrm{MH}]^{+}\left(\mathrm{C}_{17} \mathrm{H}_{30} \mathrm{NO}_{2} \mathrm{~S}\right) \mathrm{m} / \mathrm{z}$ 312.1997. Found 312.2001.

$\boldsymbol{N}$-Sulfinyl-1,3-Amino Alcohol anti-5j. General procedure $\mathrm{C}$ was followed with $55 \mathrm{mg}(0.200 \mathrm{mmol})$ of $\mathbf{2} \mathbf{j}$, and $0.50 \mathrm{~mL}$ of $\mathrm{LiBHEt}_{3}(0.50 \mathrm{mmol}, 1.00 \mathrm{M}$ in THF). Diastereoselectivity was determined by HPLC analysis; 99:1 dr (97:3 hexanes/EtOH, $1.0 \mathrm{~mL} / \mathrm{min}$ : major diastereomer, $\mathrm{t}_{\mathrm{R}}=8.2 \mathrm{~min}$; minor diastereomer, $\mathrm{t}_{\mathrm{R}}=18.3 \mathrm{~min}$ ). Column chromatography (7:3 hexanes/EtOAc) afforded $53 \mathrm{mg}(96 \%)$ of anti-5j: IR $3261,3384 \mathrm{~cm}^{-1}$; ${ }^{1} \mathrm{H}$ NMR (500 MHz) $\delta 0.87(\mathrm{~s}, 9 \mathrm{H}), 0.88(\mathrm{~d}, 6 \mathrm{H}, J=6.7 \mathrm{~Hz}), 1.22(\mathrm{~s}$, 9H), $1.54(\mathrm{ddd}, 1 \mathrm{H}, J=14.5,8.9,2.5 \mathrm{~Hz}), 1.65(\mathrm{ddd}, 1 \mathrm{H}, J=14.5,11.4,3.4 \mathrm{~Hz}), 1.71$ (dsep, $1 \mathrm{H}, J=6.7,5.6 \mathrm{~Hz}), 3.24$ (dddd, $1 \mathrm{H}, J=8.9,8.4,5.6,3.4 \mathrm{~Hz}$ ), 3.31 (d, $1 \mathrm{H}, J=$ $8.4 \mathrm{~Hz}), 3.35-3.38(\mathrm{~m}, 1 \mathrm{H}), 3.54(\mathrm{~d}, 1 \mathrm{H}, J=6.4 \mathrm{~Hz}) ;{ }^{13} \mathrm{C} \mathrm{NMR}(125 \mathrm{MHz}) \delta 18.2,19.8$, 22.8, 25.8, 33.2, 33.9, 34.9, 56.0, 59.9, 74.3. Anal. Calcd. for $\mathrm{C}_{14} \mathrm{H}_{31} \mathrm{NO}_{2} \mathrm{~S}$ : C, 60.60; $\mathrm{H}$, 11.26; N, 5.05. Found: C, 60.36; H, 11.43; N, 4.86.

$\mathrm{N}$-Sulfinyl-1,3-Amino Alcohol anti-5k. General procedure $\mathrm{C}$ was followed with $52 \mathrm{mg}(0.200 \mathrm{mmol})$ of $\mathbf{2} \mathbf{k}$, and $0.50 \mathrm{~mL}$ of $\mathrm{LiBHEt}_{3}(0.50 \mathrm{mmol}, 1.00 \mathrm{M}$ in THF$)$. Diastereoselectivity was determined by HPLC analysis; 99:1 dr (97:3 hexanes/EtOH, $1.0 \mathrm{~mL} / \mathrm{min}$ : major diastereomer, $\mathrm{t}_{\mathrm{R}}=11.2 \mathrm{~min}$; minor diastereomer, $\mathrm{t}_{\mathrm{R}}=33.8 \mathrm{~min}$ ). Column chromatography (5:5 hexanes/EtOAc) afforded $49 \mathrm{mg}(94 \%)$ of anti-5k: IR 3258, $3366 \mathrm{~cm}^{-1}$; ${ }^{1} \mathrm{H}$ NMR (500 MHz) $\delta 0.87(\mathrm{~d}, 3 \mathrm{H}, J=6.8 \mathrm{~Hz}), 0.87(\mathrm{~d}, 6 \mathrm{H}, J=6.8$ $\mathrm{Hz}), 0.93(\mathrm{~d}, 3 \mathrm{H}, J=6.7 \mathrm{~Hz}), 1.22(\mathrm{~s}, 9 \mathrm{H}), 1.53(\mathrm{ddd}, 1 \mathrm{H}, J=14.6,9.6,2.6 \mathrm{~Hz})$, 1.62-1.73 (m, 3H), 3.25-3.30 (m, 1H), 3.45 (d, 1H, $J=7.7 \mathrm{~Hz}), 3.46$ (br s, 1H), 3.72 (br s, $1 \mathrm{H}) ;{ }^{13} \mathrm{C}$ NMR $(125 \mathrm{MHz}) \delta 18.0,18.2,18.9,19.4,22.8,33.4,33.9,36.6,56.0,59.2$, 72.1; HRMS (FAB) calcd for $[\mathrm{MH}]^{+}\left(\mathrm{C}_{13} \mathrm{H}_{30} \mathrm{NO}_{2} \mathrm{~S}\right) \mathrm{m} / z$ 264.1997. Found 264.1994.

$\boldsymbol{N}$-Sulfinyl-1,3-Amino Alcohol anti-5l. General procedure $\mathrm{C}$ was followed with $49 \mathrm{mg}(0.200 \mathrm{mmol})$ of $2 \mathbf{l}$, and $0.50 \mathrm{~mL}$ of $\mathrm{LiBHEt}_{3}(0.50 \mathrm{mmol}, 1.00 \mathrm{M}$ in THF). Diastereoselectivity was determined by HPLC analysis; 98:2 dr (97:3 hexanes/EtOH, $1.0 \mathrm{~mL} / \mathrm{min}$ : major diastereomer, $\mathrm{t}_{\mathrm{R}}=15.9 \mathrm{~min}$; minor diastereomer, $\mathrm{t}_{\mathrm{R}}=50.1 \mathrm{~min}$ ). Column chromatography (5:5 hexanes/EtOAc) afforded $46 \mathrm{mg}(94 \%)$ of anti-5l: IR 3230, $3425 \mathrm{~cm}^{-1}$; ${ }^{1} \mathrm{H}$ NMR (500 MHz) $\delta 0.87(\mathrm{~d}, 3 \mathrm{H}, J=6.7 \mathrm{~Hz}), 0.88(\mathrm{~d}, 3 \mathrm{H}, J=6.7$ $\mathrm{Hz}), 0.93(\mathrm{t}, 3 \mathrm{H}, J=7.4 \mathrm{~Hz}), 1.23(\mathrm{~s}, 9 \mathrm{H}), 1.42-1.72(\mathrm{~m}, 5 \mathrm{H}), 3.28-3.34(\mathrm{~m}, 1 \mathrm{H}), 3.45$ $(\mathrm{d}, 1 \mathrm{H}, J=7.4 \mathrm{~Hz}), 3.64-3.69(\mathrm{~m}, 1 \mathrm{H}), 3.84$ (br s, $1 \mathrm{H}) ;{ }^{13} \mathrm{C}$ NMR $(125 \mathrm{MHz}) \delta 10.4$, $17.8,19.3,22.8,30.2,33.5,39.3,56.0,58.7,68.8$; HRMS (FAB) calcd for $[\mathrm{MH}]^{+}$ $\left(\mathrm{C}_{12} \mathrm{H}_{28} \mathrm{NO}_{2} \mathrm{~S}\right) \mathrm{m} / \mathrm{z} 250.1841$. Found 250.1838.

$\mathrm{N}$-Sulfinyl-1,3-Amino Alcohol anti-5m. General procedure $\mathrm{C}$ was followed with $59 \mathrm{mg}(0.200 \mathrm{mmol})$ of $\mathbf{2 m}$, and $0.50 \mathrm{~mL}$ of $\operatorname{LiBHEt}_{3}(0.50 \mathrm{mmol}, 1.00 \mathrm{M}$ in THF). Diastereoselectivity was determined by HPLC analysis; 92:8 dr (94:6 hexanes/EtOH, $1.0 \mathrm{~mL} / \mathrm{min}$ : major diastereomer, $\mathrm{t}_{\mathrm{R}}=6.7 \mathrm{~min}$; minor diastereomer, $\mathrm{t}_{\mathrm{R}}=25.0 \mathrm{~min}$ ). 
Column chromatography (6:4 hexanes/EtOAc) afforded $50 \mathrm{mg}(85 \%)$ of anti-5m: IR $3215,3401 \mathrm{~cm}^{-1}$; ${ }^{1} \mathrm{H}$ NMR (500 MHz) $\delta 0.88(\mathrm{~d}, 3 \mathrm{H}, J=6.8 \mathrm{~Hz}), 0.90$ (d, 3H, $J=6.8$ $\mathrm{Hz}), 1.26$ (s, 9H), 1.72-1.80 (m, 2H), 1.96 (ddd, 1H, $J=14.7,10.6,3.0 \mathrm{~Hz}), 3.36-3.41$ (m, 1H), 3.55 (d, 1H, $J=7.9 \mathrm{~Hz}), 4.36$ (br s, 1H), 4.91 (dd, 1H, $J=10.3,1.8 \mathrm{~Hz}$ ), 7.21-7.39 (m, 5H); ${ }^{13} \mathrm{C}$ NMR (125 MHz) $\delta 17.9,19.2,22.9,33.3,42.2,56.2,59.2,69.7$, 125.6, 127.0, 128.3, 144.8; HRMS (FAB) calcd for $[\mathrm{MH}]^{+}\left(\mathrm{C}_{16} \mathrm{H}_{28} \mathrm{NO}_{2} \mathrm{~S}\right) m / z 298.1841$. Found 298.1840.

N-Sulfinyl-1,3-Amino Alcohol anti-5n. General procedure $\mathrm{C}$ was followed with $52 \mathrm{mg}(0.200 \mathrm{mmol})$ of $\mathbf{2 n}$, and $0.50 \mathrm{~mL}$ of $\mathrm{LiBHEt}_{3}(0.50 \mathrm{mmol}, 1.00 \mathrm{M}$ in THF). Diastereoselectivity was determined by HPLC analysis; 97:3 dr (97:3 hexanes/EtOH, $1.0 \mathrm{~mL} / \mathrm{min}$ : major diastereomer, $\mathrm{t}_{\mathrm{R}}=11.5 \mathrm{~min}$; minor diastereomer, $\mathrm{t}_{\mathrm{R}}=21.7 \mathrm{~min}$ ). Column chromatography (7:3 hexanes/EtOAc) afforded $47 \mathrm{mg}$ (90\%) of anti-5n: IR 3253, $3458 \mathrm{~cm}^{-1}$; ${ }^{1} \mathrm{H}$ NMR (500 MHz) $\delta 0.87(\mathrm{~s}, 9 \mathrm{H}), 0.93(\mathrm{t}, 3 \mathrm{H}, J=7.4 \mathrm{~Hz}), 1.22(\mathrm{~s}$, 9H), $1.48-1.58$ (m, 3H), 1.82 (ddd, $1 \mathrm{H}, J=14.8,11.4,3.6 \mathrm{~Hz}), 3.30-3.43$ (m, 3H), 3.58 $(\mathrm{d}, 1 \mathrm{H}, J=9.0 \mathrm{~Hz}) ;{ }^{13} \mathrm{C}$ NMR $(125 \mathrm{MHz}) \delta 11.0,22.7,25.7,29.4,34.9,36.0,55.8,57.1$, 74.8. Anal. Calcd. for $\mathrm{C}_{13} \mathrm{H}_{29} \mathrm{NO}_{2} \mathrm{~S}$ : C, 59.27; H, 11.10; N, 5.32. Found: C, 59.23; H, $11.46 ; \mathrm{N}, 5.17$.

$\boldsymbol{N}$-Sulfinyl-1,3-Amino Alcohol anti-5o. General procedure $\mathrm{C}$ was followed with $49 \mathrm{mg}(0.200 \mathrm{mmol})$ of $\mathbf{2 0}$, and $0.50 \mathrm{~mL}$ of $\mathrm{LiBHEt}_{3}(0.50 \mathrm{mmol}, 1.00 \mathrm{M}$ in THF). Diastereoselectivity was determined by HPLC analysis; 95:5 dr (97:3 hexanes/EtOH, $1.0 \mathrm{~mL} / \mathrm{min}$ : major diastereomer, $\mathrm{t}_{\mathrm{R}}=15.7 \mathrm{~min}$; minor diastereomer, $\mathrm{t}_{\mathrm{R}}=37.6 \mathrm{~min}$ ). Column chromatography (6:4 hexanes/EtOAc) afforded $46 \mathrm{mg}(94 \%)$ of anti-5o: IR $3259,3370 \mathrm{~cm}^{-1}$; ${ }^{1} \mathrm{H}$ NMR $(500 \mathrm{MHz}) \delta 0.87(\mathrm{~d}, 3 \mathrm{H}, J=6.8 \mathrm{~Hz}), 0.91(\mathrm{~d}, 3 \mathrm{H}, J=6.7$ $\mathrm{Hz}), 0.91(\mathrm{t}, 3 \mathrm{H}, J=7.4 \mathrm{~Hz}), 1.21(\mathrm{~s}, 9 \mathrm{H}), 1.46-1.56(\mathrm{~m}, 3 \mathrm{H}), 1.63(\mathrm{dtt}, 1 \mathrm{H}, J=5.9,6.7$, $6.8 \mathrm{~Hz}), 1.80$ (ddd, $1 \mathrm{H}, J=14.7,10.5,3.5 \mathrm{~Hz}), 3.33-3.39(\mathrm{~m}, 1 \mathrm{H}), 3.45-3.54(\mathrm{~m}, 2 \mathrm{H})$, $3.69(\mathrm{~d}, 1 \mathrm{H}, J=8.2 \mathrm{~Hz}) ;{ }^{13} \mathrm{C}$ NMR $(125 \mathrm{MHz}) \delta 10.7,17.9,18.7,22.7,29.7,34.1,38.8$, 55.8, 56.5, 72.4; HRMS (FAB) calcd for $[\mathrm{MH}]^{+}\left(\mathrm{C}_{12} \mathrm{H}_{28} \mathrm{NO}_{2} \mathrm{~S}\right) \mathrm{m} / z$ 250.1841. Found 250.1836 .

$\mathrm{N}$-Sulfinyl-1,3-Amino Alcohol anti-5p. General procedure $\mathrm{C}$ was followed with $70 \mathrm{mg}(0.300 \mathrm{mmol})$ of $\mathbf{2 p}$, and $0.75 \mathrm{~mL}$ of $\mathrm{LiBHEt}_{3}(0.75 \mathrm{mmol}, 1.00 \mathrm{M}$ in THF $)$. Diastereoselectivity was determined by HPLC analysis; 93:7 dr (94:6 hexanes/EtOH, $1.0 \mathrm{~mL} / \mathrm{min}$ : major diastereomer, $\mathrm{t}_{\mathrm{R}}=9.5 \mathrm{~min}$; minor diastereomer, $\mathrm{t}_{\mathrm{R}}=20.3 \mathrm{~min}$ ). Column chromatography (5:5 hexanes/EtOAc) afforded $55 \mathrm{mg}(78 \%)$ of anti-5p: IR 3244, $3390 \mathrm{~cm}^{-1}$; ${ }^{1} \mathrm{H}$ NMR (500 MHz) $\delta 0.92(\mathrm{t}, 3 \mathrm{H}, J=7.4 \mathrm{~Hz}), 0.93$ (t, 3H, $J=7.4$ $\mathrm{Hz}), 1.22$ (s, 9H), 1.42-1.56 (m, 5H), $1.76(\mathrm{ddd}, 1 \mathrm{H}, J=14.7,10.1,3.5 \mathrm{~Hz}), 3.35-3.42$ (m, 1H), 3.50 (br s, 1H), $3.66(\mathrm{~d}, 1 \mathrm{H}, J=7.8 \mathrm{~Hz}), 3.68-3.73(\mathrm{~m}, 1 \mathrm{H}) ;{ }^{13} \mathrm{C}$ NMR $(125$ 
MHz) $\delta 10.2,10.6,22.7,29.9,30.5,41.8,55.8,56.0,69.1$; HRMS (FAB) calcd for $[\mathrm{MH}]^{+}\left(\mathrm{C}_{11} \mathrm{H}_{26} \mathrm{NO}_{2} \mathrm{~S}\right) m / z$ 236.1684. Found 236.1678.

$\mathrm{N}$-Sulfinyl-1,3-Amino Alcohol anti-5q. General procedure $\mathrm{C}$ was followed with $56 \mathrm{mg}(0.200 \mathrm{mmol})$ of $\mathbf{2 q}$, and $0.50 \mathrm{~mL}$ of $\mathrm{LiBHEt}_{3}(0.50 \mathrm{mmol}, 1.00 \mathrm{M}$ in THF). Diastereoselectivity was determined by HPLC analysis; 88:12 dr (94:6 hexanes/EtOH, $1.0 \mathrm{~mL} / \mathrm{min}$ : major diastereomer, $\mathrm{t}_{\mathrm{R}}=8.6 \mathrm{~min}$; minor diastereomer, $\mathrm{t}_{\mathrm{R}}=25.0 \mathrm{~min}$ ). Column chromatography (6:4 hexanes/EtOAc) afforded $45 \mathrm{mg}(80 \%)$ of anti-5q: IR $3191,3346 \mathrm{~cm}^{-1}$; ${ }^{1} \mathrm{H}$ NMR (500 MHz) $\delta 0.94$ (t, 3H, $\left.J=7.4 \mathrm{~Hz}\right), 1.25(\mathrm{~s}, 9 \mathrm{H}), 1.53-1.60$ (m, 2H), 1.77 (ddd, 1H, $J=14.8,8.4,3.0 \mathrm{~Hz}$ ), 2.10 (ddd, 1H, $J=14.8,10.5,3.4 \mathrm{~Hz}$ ), 3.40-3.47 (m, 1H), 3.72 (d, 1H, $J=8.1 \mathrm{~Hz}), 4.04$ (br s, 1H), 4.93 (dd, 1H, $J=10.3,2.2$ $\mathrm{Hz}), 7.22-7.37(\mathrm{~m}, 5 \mathrm{H}) ;{ }^{13} \mathrm{C} \mathrm{NMR}(125 \mathrm{MHz}) \delta 10.6,22.7,29.6,44.4,55.9,56.2,70.2$, 125.6, 127.1, 128.3, 144.8. Anal. Calcd. for $\mathrm{C}_{15} \mathrm{H}_{25} \mathrm{NO}_{2} \mathrm{~S}: \mathrm{C}, 63.56 ; \mathrm{H}, 8.89 ; \mathrm{N}, 4.94$. Found: C, 63.25; H, 9.19; N, 4.76.

Sulfinyl Imine 7. A mixture of $1.52 \mathrm{~g}(12.5 \mathrm{mmol})$ of tert-butanesulfinamide, $2.37 \mathrm{~g}$ of ketone $6(15.0 \mathrm{mmol})$, and $6.0 \mathrm{~g}$ of Ti(OEt $)_{4}(\sim 25.0 \mathrm{mmol})$ in $25 \mathrm{~mL}$ of THF was heated to reflux for $15 \mathrm{~h}$ and then was cooled to room temperature. The reaction mixture was poured into $25 \mathrm{~mL}$ of brine while rapidly stirring, and the resulting mixture was filtered through a plug of Celite. The filter cake was washed with EtOAc, and the combined filtrate was transferred to a separatory funnel where the organic layer was washed with brine. The brine layer was extracted once with EtOAc, and the combined organic portions were dried over $\mathrm{Na}_{2} \mathrm{SO}_{4}$, filtered and concentrated. Column chromatography (4:6 hexanes/EtOAc) of the crude material afforded $3.21 \mathrm{~g}(82 \%)$ of 7 as a mixture of $E / Z$ isomers $\left(8: 1\right.$ by ${ }^{1} \mathrm{H}$ NMR in $\left.\mathrm{CDCl}_{3}\right)$ : IR $1621 \mathrm{~cm}^{-1}$; ${ }^{1} \mathrm{H}$ NMR (500 MHz) $\delta 1.25(\mathrm{~s}, 9 \mathrm{H}), 1.66-1.77(\mathrm{~m}, 4 \mathrm{H}), 2.31(\mathrm{~s}, 3 \mathrm{H}), 2.39-2.51(\mathrm{~m}, 2 \mathrm{H}), 3.81-3.98(\mathrm{~m}$, $4 \mathrm{H}), 4.85(\mathrm{t}, 1 \mathrm{H}, J=4.4 \mathrm{~Hz}) ;{ }^{13} \mathrm{C}$ NMR $(125 \mathrm{MHz}) \delta 19.8,22.1,23.0,33.1,43.0,56.2$, 64.9, 104.2, 185.0; HRMS (FAB) calcd for $[\mathrm{MH}]^{+}\left(\mathrm{C}_{12} \mathrm{H}_{24} \mathrm{NO}_{3} \mathrm{~S}\right) \mathrm{m} / z 262.1477$. Found 262.1473.

$\beta$-Hydroxy Sulfinyl Imine 8. To a solution of $485 \mathrm{mg}$ of $i-\operatorname{Pr}_{2} \mathrm{NH}$ (4.79 $\mathrm{mmol}$ ) in $20 \mathrm{~mL}$ of THF was added $2.0 \mathrm{~mL}$ of a $2.20 \mathrm{M}$ solution of butyllithium in pentane (4.40 equiv) at $0{ }^{\circ} \mathrm{C}$ and the mixture was stirred for $30 \mathrm{~min}$. The solution was then cooled to $-78{ }^{\circ} \mathrm{C}$ and $1.05 \mathrm{~g}$ of tert-butanesulfinyl imine 7 (4.02 mmol) was added. After the reaction solution was stirred for $45 \mathrm{~min}$ at $-78{ }^{\circ} \mathrm{C}, 1.47 \mathrm{~g}$ of $\mathrm{MgBr}_{2}$ (7.98 equiv) was added. The reaction mixture was stirred for $45 \mathrm{~min}$, and $317 \mathrm{mg}$ of butyraldehyde $(4.40 \mathrm{mmol})$ was added to the solution. Then, the mixture was stirred for $20 \mathrm{~h}$ at $-78{ }^{\circ} \mathrm{C}$. To the reaction mixture was added $2 \mathrm{~N}$ AcOH/THF ( 5 equiv), which was cooled to $-78{ }^{\circ} \mathrm{C}$ before the addition. Brine and saturated aqueous $\mathrm{NaHCO}_{3}$ 
solution was then added to the mixture with stirring. The organic layer was collected and the brine layer was extracted with EtOAc $(2 \mathrm{x})$. The combined organic portions were quickly dried, filtered, and concentrated. Diastereoselectivity was determined by HPLC analysis; 82:18 dr (94:6 hexanes $/ i-\mathrm{PrOH}, 1.0 \mathrm{~mL} / \mathrm{min}$ : major diastereomer, $\mathrm{t}_{\mathrm{R}}=$ $17.8 \mathrm{~min}$; minor diastereomer, $\left.t_{\mathrm{R}}=24.8 \mathrm{~min}\right)$. Column chromatography $(6: 4$ hexanes/EtOAc) of the crude material afforded $677 \mathrm{mg}(51 \%)$ of 8 as a mixture of $E / Z$ isomers (13:1 by ${ }^{1} \mathrm{H}$ NMR in $\mathrm{CDCl}_{3}$ ): IR $1623,3348 \mathrm{~cm}^{-1} ;{ }^{1} \mathrm{H}$ NMR (500 MHz) $\delta 0.91$ $(\mathrm{t}, 3 \mathrm{H}, J=6.9 \mathrm{~Hz}), 1.24(\mathrm{~s}, 9 \mathrm{H}), 1.31-1.51(\mathrm{~m}, 4 \mathrm{H}), 1.62-1.80(\mathrm{~m}, 4 \mathrm{H}), 2.38-2.65(\mathrm{~m}$, $3 \mathrm{H}), 3.09(\mathrm{~m}, 1 \mathrm{H}), 3.72-3.79(\mathrm{~m}, 1 \mathrm{H}), 3.82-3.97(\mathrm{~m}, 4 \mathrm{H}), 4.08(\mathrm{~d}, 1 \mathrm{H}, J=9.3 \mathrm{~Hz}), 4.84$ $(\mathrm{t}, 1 \mathrm{H}, J=4.4 \mathrm{~Hz}) ;{ }^{13} \mathrm{C}$ NMR $(125 \mathrm{MHz}) \delta 14.0,18.7,19.7,22.4,33.0,40.6,41.4,45.1$, 57.7, 64.8, 67.3, 104.3, 184.6; HRMS (FAB) calcd for $[\mathrm{MH}]^{+}\left(\mathrm{C}_{16} \mathrm{H}_{32} \mathrm{NO}_{4} \mathrm{~S}\right) \mathrm{m} / \mathrm{z}$ 334.2052. Found 334.2053.

$\mathrm{N}$-Sulfinyl-1,3-amino Alcohol syn-9. To a solution of $50 \mathrm{mg}$ of $\beta$-hydroxy sulfinyl imine $8(0.150 \mathrm{mmol}, 1.0$ equiv) in $0.75 \mathrm{~mL}$ of THF was added $45 \mathrm{mg}$ of catecholborane ( $0.375 \mathrm{mmol}, 2.5$ equiv), and the mixture was stirred for $3 \mathrm{~h}$ at $-48{ }^{\circ} \mathrm{C}$. To this mixture was added $\mathrm{MeOH}$ and a saturated solution of sodium potassium tartrate. The resulting mixture was stirred for $10 \mathrm{~min}$, washed with brine, and the brine layer was extracted with EtOAc (2x). The combined organic portions were dried, filtered, and concentrated. Diastereoselectivity was determined by HPLC analysis; 93:7 dr (90:10 hexanes $/ \mathrm{EtOH}, 1.0 \mathrm{~mL} / \mathrm{min}$ : major diastereomer, $\mathrm{t}_{\mathrm{R}}=30.4 \mathrm{~min}$; minor diastereomer, $\mathrm{t}_{\mathrm{R}}$ $=13.6 \mathrm{~min})$. Column chromatography $(19: 1 \mathrm{EtOAc} / \mathrm{MeOH})$ of the crude material afforded $36 \mathrm{mg}$ (72\%) of syn-9: IR 3288, $3395 \mathrm{~cm}^{-1}$; ${ }^{1} \mathrm{H}$ NMR $(500 \mathrm{MHz}) \delta 0.88(\mathrm{t}, 3 \mathrm{H}$, $J=7.0 \mathrm{~Hz}), 1.18(\mathrm{~s}, 9 \mathrm{H}), 1.25-1.79(\mathrm{~m}, 12 \mathrm{H}), 3.11($ br s, $1 \mathrm{H}), 3.30-3.36(\mathrm{~m}, 1 \mathrm{H})$, 3.65-3.70 (m, 1H), 3.77-3.96 (m, 4H), $3.99(\mathrm{~d}, 1 \mathrm{H}, J=5.5 \mathrm{~Hz}), 4.81(\mathrm{t}, 1 \mathrm{H}, J=4.7 \mathrm{~Hz})$;

${ }^{13} \mathrm{C}$ NMR (125 MHz) $\delta 14.0,18.4,20.3,22.5,33.3,35.3,40.6,42.9,55.8,56.5,64.7$, 64.7, 71.2, 104.2; HRMS (FAB) calcd for $[\mathrm{MH}]^{+}\left(\mathrm{C}_{16} \mathrm{H}_{34} \mathrm{NO}_{4} \mathrm{~S}\right) \mathrm{m} / z$ 336.2209. Found 336.2212 .

$\mathrm{N}$-Sulfinyl-1,3-amino Alcohol anti-9. To a $-78{ }^{\circ} \mathrm{C}$ solution of $133 \mathrm{mg}$ of $\beta$-hydroxy $N$-sulfinyl imine 8 (0.399 mmol, 1.00 equiv) in $2 \mathrm{~mL}$ of THF was added 1 $\mathrm{mL}$ of a $1.0 \mathrm{M}$ solution of $\mathrm{LiBHEt}_{3}(1.00 \mathrm{mmol}, 2.51$ equiv) and the resulting solution was stirred for $3 \mathrm{~h}$. To this solution was added $1.00 \mathrm{~mL}$ of a saturated aqueous solution of $\mathrm{NH}_{4} \mathrm{Cl}$. The resulting mixture was gradually warmed to $\mathrm{rt}$ with stirring, washed with brine, and the brine layer was extracted with EtOAc $(2 x)$. The combined organic portions were dried, filtered, and concentrated. Diastereoselectivity was determined by HPLC analysis; 91:9 dr (90:10 hexanes/EtOH, $1.0 \mathrm{~mL} / \mathrm{min}$ : major diastereomer, $\mathrm{t}_{\mathrm{R}}=$ $12.5 \mathrm{~min}$; minor diastereomer, $\left.t_{\mathrm{R}}=29.9 \mathrm{~min}\right)$. Column chromatography $(3: 7$ 
hexane/EtOAc) of the crude material afforded $101 \mathrm{mg}$ (75\%) of anti-9: IR 3252, 3402 $\mathrm{cm}^{-1} ;{ }^{1} \mathrm{H}$ NMR $(500 \mathrm{MHz}) \delta 0.86(\mathrm{t}, 3 \mathrm{H}, J=7.1 \mathrm{~Hz}), 1.17(\mathrm{~s}, 9 \mathrm{H}), 1.25-1.66(\mathrm{~m}, 11 \mathrm{H})$, 1.73 (ddd, $1 \mathrm{H}, J=14.7,10.1,3.5), 2.69$ (br s, $1 \mathrm{H}), 3.39-3.45(\mathrm{~m}, 1 \mathrm{H}), 3.60$ (d, $1 \mathrm{H}, J=$ $6.0 \mathrm{~Hz}), 3.73-3.94(\mathrm{~m}, 5 \mathrm{H}), 4.78(\mathrm{t}, 1 \mathrm{H}, J=4.7 \mathrm{~Hz}) ;{ }^{13} \mathrm{C} \mathrm{NMR}(125 \mathrm{MHz}) \delta 14.0,18.9$, 20.4, 22.6, 33.4, 36.6, 39.8, 42.3, 54.4, 55.7, 64.7, 64.7, 67.5, 104.3; HRMS (FAB) calcd for $[\mathrm{MH}]^{+}\left(\mathrm{C}_{16} \mathrm{H}_{34} \mathrm{NO}_{4} \mathrm{~S}\right) \mathrm{m} / \mathrm{z}$ 336.2209. Found 336.2215.

(-)-8-Epihalosaline (syn-10). A mixture of $70 \mathrm{mg}$ of syn-9 (0.209 mmol, 1.0 equiv) and $4.7 \mathrm{mg}$ of $\mathrm{PtO}_{2}(0.021 \mathrm{mmol})$ in $11 \mathrm{~mL}$ of $\mathrm{TFA} / \mathrm{EtOH} / \mathrm{H}_{2} \mathrm{O}(5: 5: 1)$ was stirred under a hydrogen atomosphere at room temperature for $15 \mathrm{~h}$. An aqueous solution of $\mathrm{KOH}$ was added to the resulting mixture until the $\mathrm{pH}$ was 14 , and then the mixture was extracted with $\mathrm{CH}_{2} \mathrm{Cl}_{2}(5 \mathrm{x})$. The combined organic portions were dried over $\mathrm{Na}_{2} \mathrm{SO}_{4}$, filtered and concentrated. Column chromatography (7:1.4:91.6 $\mathrm{MeOH} / 15 \mathrm{~N} \mathrm{NH} 4 \mathrm{OH}$ aq/ $\mathrm{CH}_{2} \mathrm{Cl}_{2}$ ) of the crude material afforded $26 \mathrm{mg}(73 \%)$ of (-)-8-epihalosaline: $[\alpha]_{\mathrm{D}}{ }^{23}-7.9(\mathrm{c} 1.5, \mathrm{MeOH})\left[\right.$ lit. value. $\left.{ }^{7}[\alpha]_{\mathrm{D}}{ }^{23}-8(\mathrm{c} 3.7, \mathrm{MeOH})\right] ;{ }^{1} \mathrm{H}$ NMR $(500 \mathrm{MHz}) \delta 0.90(\mathrm{t}, 3 \mathrm{H}, J=7.0 \mathrm{~Hz}), 1.05-1.64(\mathrm{~m}, 11 \mathrm{H}), 1.78-1.83(\mathrm{~m}, 1 \mathrm{H})$, $2.34(\mathrm{~s}, 1 \mathrm{H}), \quad 2.55-2.61(\mathrm{~m}, 1 \mathrm{H}), 2.68-2.73(\mathrm{~m}, 1 \mathrm{H}), 3.01-3.04(\mathrm{~m}, 1 \mathrm{H}), 3.13(\mathrm{br} \mathrm{s}, 1 \mathrm{H})$, 3.79-3.84 (m, 1H); ${ }^{13} \mathrm{C}$ NMR (125 MHz) $\delta 14.1,18.6,24.5,27.2,34.3,40.3,42.4,45.9$, 58.3, 72.8; HRMS (EI) calcd for $[\mathrm{M}]^{+}\left(\mathrm{C}_{10} \mathrm{H}_{21} \mathrm{NO}\right) \mathrm{m} / z$ 171.1623. Found 171.1622.

(-)-Halosaline (anti-10). A mixture of $70 \mathrm{mg}$ of anti-9 $(0.209 \mathrm{mmol}, 1.0$ equiv) and $4.7 \mathrm{mg}$ of $\mathrm{PtO}_{2}(0.021 \mathrm{mmol})$ in $11 \mathrm{~mL}$ of $\mathrm{TFA} / \mathrm{EtOH} / \mathrm{H}_{2} \mathrm{O}$ (5:5:1) was stirred under a hydrogen atomosphere at room temperature for $15 \mathrm{~h}$. An aqueous solution of $\mathrm{KOH}$ was added to the resulting mixture until the $\mathrm{pH}$ was 14 , and then the mixture was extracted with $\mathrm{CH}_{2} \mathrm{Cl}_{2}(5 \mathrm{x})$. The combined organic portions were dried over $\mathrm{Na}_{2} \mathrm{SO}_{4}$, filtered and concentrated. Column chromatography (15:2.5:82.5 $\mathrm{MeOH} / 15 \mathrm{~N} \mathrm{NH} 4 \mathrm{OH} \mathrm{aq} / \mathrm{CH}_{2} \mathrm{Cl}_{2}$ ) of the crude material afforded $29 \mathrm{mg}(81 \%)$ of (-)-halosaline: $[\alpha]_{\mathrm{D}}{ }^{23}-18.2($ c $0.6,95 \% \mathrm{EtOH})\left[\right.$ lit. value. ${ }^{8}[\alpha]_{\mathrm{D}}{ }^{20}-19.5$ (c $0.6,95 \%$ EtOH)]; ${ }^{1} \mathrm{H}$ NMR (500 MHz) $\delta 0.90(\mathrm{t}, 3 \mathrm{H}, J=7.1 \mathrm{~Hz}), 1.26-1.59(\mathrm{~m}, 11 \mathrm{H}), 1.78-1.80$ $(\mathrm{m}, 1 \mathrm{H}), 2.53-2.58(\mathrm{~m}, 1 \mathrm{H}), 2.85-2.89(\mathrm{~m}, 1 \mathrm{H}), 3.02-3.06(\mathrm{~m}, 1 \mathrm{H}), 3.43(\mathrm{~s}, 1 \mathrm{H}), 3.51$ (br $\mathrm{s}, 1 \mathrm{H}), 3.85-3.90(\mathrm{~m}, 1 \mathrm{H}) ;{ }^{13} \mathrm{C} \mathrm{NMR}(125 \mathrm{MHz}) \delta 14.1,19.0,24.6,25.9,31.3,40.0$, 41.9, 46.8, 54.7, 68.6; HRMS (FAB) calcd for $[\mathrm{MH}]^{+}\left(\mathrm{C}_{10} \mathrm{H}_{22} \mathrm{NO}\right) \mathrm{m} / z 172.1701$. Found 172.1705. 
Table S1. Crystal Data and Structural Refinement for anti-5h

\begin{tabular}{|c|c|}
\hline & anti-5h \\
\hline Empirical Formula & $\mathrm{SO}_{2} \mathrm{NC}_{13} \mathrm{H}_{29}$ \\
\hline Formula Weight & 263.44 \\
\hline Temperature $\left({ }^{\circ} \mathrm{C}\right)$ & $-139(1)$ \\
\hline Crystal Color, Habit & colorless, block \\
\hline Crystal Dimensions & $0.20 \times 0.19 \times 0.11 \mathrm{~mm}$ \\
\hline Crystal System & orthorhombic \\
\hline $\mathrm{a}(\AA)$ & $9.389(1)$ \\
\hline $\mathrm{b}(\AA)$ & $10.800(1)$ \\
\hline $\mathrm{c}(\AA)$ & $16.087(2)$ \\
\hline$V\left(\AA^{3}\right)$ & $1631.3(3)$ \\
\hline Space Group & $\mathrm{P} 2{ }_{1} 2_{1} 2_{1}(\# 19)$ \\
\hline$Z$ values & 4 \\
\hline $\mathrm{D}_{\text {calc }}$ & $1.073 \mathrm{~g} / \mathrm{cm}^{3}$ \\
\hline $\mathrm{F}_{000}$ & 584.00 \\
\hline$\mu(\operatorname{MoK} \alpha)$ & $1.92 \mathrm{~cm}^{-1}$ \\
\hline Diffractometer & Bruker SMART CCD \\
\hline Radiation & $\begin{array}{l}\text { MoK } \alpha(\lambda=0.71069 \AA) \\
\text { graphite monochromated }\end{array}$ \\
\hline Detector Position & $60.00 \mathrm{~mm}$ \\
\hline Exposure Time & 10.0 seconds per frame \\
\hline Scan Type & $\omega(0.3$ degrees per frame $)$ \\
\hline $2 \theta_{\max }$ & $49.4^{\circ}$ \\
\hline \multirow[t]{2}{*}{ No. Reflections Measured } & Total: 7456 \\
\hline & Unique: $1813\left(\mathrm{R}_{\text {int }}=0.081\right)$ \\
\hline Transmission Factors & $\mathrm{T}_{\max }=1.00, \mathrm{~T}_{\min }=0.54$ \\
\hline Structure Solution & Direct Methods (SIR97) \\
\hline Refinement & Full-matrix least-squares \\
\hline p-factor & 0.0300 \\
\hline Anomalous Dispersion & All non-hydrogen atoms \\
\hline No. Observation $(\mathrm{I}>3.00 \sigma(\mathrm{I}))$ & 1519 \\
\hline No. Variables & 154 \\
\hline Reflection/Parameter Ratio & 9.86 \\
\hline Residual: $\mathrm{R} ; \mathrm{R}_{\mathrm{w}} ; \mathrm{R}_{\mathrm{all}}^{\mathrm{a}}$ & $0.052 ; 0.055 ; 0.108$ \\
\hline Goodness of Fit Indicator $^{\mathrm{b}}$ & 1.71 \\
\hline Max Shift/Error in Final Cycle & 0.01 \\
\hline Maximum peak in Final Diff. Map & $0.29 e^{-} / \AA^{3}$ \\
\hline Minimum peak in Final Diff. Map & $-0.25 e^{-} / \AA^{3}$ \\
\hline
\end{tabular}

${ }^{\mathrm{a}} \mathrm{R}=\Sigma|| F_{o}|-| F_{c}|| \Sigma\left|F_{o}\right|, \mathrm{R}_{\mathrm{w}}=\left[\left(\Sigma \mathrm{w}\left(\left|F_{o}\right|-\left|F_{c}\right|\right)^{2} / \Sigma \mathrm{w} F_{o}{ }^{2}\right]^{1 / 2}\right.$

${ }^{\mathrm{b}}$ Goodness of Fit $=\left[\left(\Sigma \mathrm{w}\left(\left|F_{o}\right|-\left|F_{c}\right|\right)^{2} /\left(\mathrm{N}_{\mathrm{obs}}-\mathrm{N}_{\text {parameters }}\right)\right)^{1 / 2}\right.$ 
Table S2. Atomic Coordinates and B(eq) Values for anti-5h

\begin{tabular}{|c|c|c|c|c|}
\hline atom & $\mathrm{x}$ & $\mathrm{y}$ & $\mathrm{z}$ & $\mathrm{B}(\mathrm{eq})$ \\
\hline$\overline{\mathrm{S}(1)}$ & $-0.0547(2)$ & $0.1302(1)$ & $0.13812(9)$ & $2.41(3)$ \\
\hline $\mathrm{O}(1)$ & $-0.1017(3)$ & $0.2637(3)$ & $0.1369(2)$ & $2.94(10)$ \\
\hline $\mathrm{O}(2)$ & $-0.1370(4)$ & $0.3302(3)$ & $-0.0288(2)$ & $2.33(10)$ \\
\hline $\mathrm{N}(1)$ & $0.0517(5)$ & $0.0968(4)$ & $0.0589(2)$ & $1.7(1)$ \\
\hline $\mathrm{C}(1)$ & $0.0741(6)$ & $0.1220(5)$ & $0.2237(3)$ & $2.1(1)$ \\
\hline$C(2)$ & $-0.0169(6)$ & $0.1258(6)$ & $0.3017(3)$ & $3.3(2)$ \\
\hline $\mathrm{C}(3)$ & $0.1723(6)$ & $0.2328(5)$ & $0.2205(3)$ & $2.4(2)$ \\
\hline$C(4)$ & $0.1550(7)$ & $-0.0008(5)$ & $0.2177(3)$ & $2.9(2)$ \\
\hline$C(5)$ & $-0.0186(5)$ & $0.0759(5)$ & $-0.0211(3)$ & $1.9(1)$ \\
\hline$C(6)$ & $0.0250(5)$ & $0.1739(5)$ & $-0.0844(3)$ & $1.8(1)$ \\
\hline$C(7)$ & $0.0056(6)$ & $0.3066(5)$ & $-0.0573(4)$ & $2.1(1)$ \\
\hline $\mathrm{C}(8)$ & $0.0371(6)$ & $0.3956(5)$ & $-0.1277(4)$ & $2.9(2)$ \\
\hline $\mathrm{C}(9)$ & $0.0327(8)$ & $0.5320(6)$ & $-0.1032(4)$ & $4.7(2)$ \\
\hline $\mathrm{C}(10)$ & $0.0039(6)$ & $-0.0606(5)$ & $-0.0503(4)$ & $2.2(2)$ \\
\hline $\mathrm{C}(11)$ & $-0.0650(7)$ & $-0.1455(5)$ & $0.0121(4)$ & $3.9(2)$ \\
\hline$C(12)$ & $0.1627(7)$ & $-0.0904(5)$ & $-0.0559(4)$ & $3.1(2)$ \\
\hline C(13) & $-0.0645(7)$ & $-0.0774(5)$ & $-0.1358(4)$ & $4.0(2)$ \\
\hline $\mathrm{H}(1)$ & -0.0724 & 0.1993 & 0.3018 & 3.9346 \\
\hline $\mathrm{H}(2)$ & -0.0780 & 0.0557 & 0.3028 & 3.9346 \\
\hline $\mathrm{H}(3)$ & 0.0430 & 0.1247 & 0.3493 & 3.9346 \\
\hline $\mathrm{H}(4)$ & 0.2347 & 0.2312 & 0.2670 & 2.8925 \\
\hline $\mathrm{H}(5)$ & 0.2266 & 0.2303 & 0.1707 & 2.8925 \\
\hline $\mathrm{H}(6)$ & 0.1175 & 0.3067 & 0.2218 & 2.8925 \\
\hline $\mathrm{H}(7)$ & 0.0889 & -0.0674 & 0.2174 & 3.5107 \\
\hline $\mathrm{H}(8)$ & 0.2091 & -0.0021 & 0.1679 & 3.5107 \\
\hline $\mathrm{H}(9)$ & 0.2169 & -0.0088 & 0.2641 & 3.5107 \\
\hline $\mathrm{H}(10)$ & -0.1179 & 0.0860 & -0.0119 & 2.2240 \\
\hline $\mathrm{H}(11)$ & 0.1230 & 0.1620 & -0.0968 & 2.1702 \\
\hline $\mathrm{H}(12)$ & -0.0300 & 0.1615 & -0.1332 & 2.1702 \\
\hline $\mathrm{H}(13)$ & 0.0698 & 0.3232 & -0.0130 & 2.4780 \\
\hline $\mathrm{H}(14)$ & 0.1294 & 0.3777 & -0.1486 & 3.5180 \\
\hline $\mathrm{H}(15)$ & -0.0314 & 0.3825 & -0.1703 & 3.5180 \\
\hline $\mathrm{H}(16)$ & 0.1043 & 0.5480 & -0.0629 & 5.6654 \\
\hline $\mathrm{H}(17)$ & 0.0494 & 0.5818 & -0.1509 & 5.6654 \\
\hline $\mathrm{H}(18)$ & -0.0581 & 0.5512 & -0.0806 & 5.6654 \\
\hline $\mathrm{H}(19)$ & -0.1516 & -0.1097 & 0.0311 & 4.7173 \\
\hline $\mathrm{H}(20)$ & -0.0843 & -0.2231 & -0.0132 & 4.7173 \\
\hline $\mathrm{H}(21)$ & -0.0026 & -0.1571 & 0.0580 & 4.7173 \\
\hline $\mathrm{H}(22)$ & 0.1885 & -0.1440 & -0.0116 & 3.6893 \\
\hline $\mathrm{H}(23)$ & 0.1821 & -0.0699 & -0.1075 & 3.6893 \\
\hline $\mathrm{H}(24)$ & 0.2162 & -0.0160 & -0.0523 & 3.6893 \\
\hline $\mathrm{H}(25)$ & -0.0748 & 0.0010 & -0.1618 & 4.7802 \\
\hline $\mathrm{H}(26)$ & -0.0055 & -0.1289 & -0.1691 & 4.7802 \\
\hline $\mathrm{H}(27)$ & -0.1553 & -0.1149 & -0.1296 & 4.7802 \\
\hline $\mathrm{H}(28)$ & 0.1403 & 0.1398 & 0.0552 & 4.0680 \\
\hline $\mathrm{H}(29)$ & -0.1607 & 0.2796 & 0.0033 & 4.0680 \\
\hline
\end{tabular}


Table S3. Anisotropic Displacement Parameters for anti-5h

\begin{tabular}{lllllll}
\hline atom & $\mathrm{U} 11$ & $\mathrm{U} 22$ & $\mathrm{U} 33$ & $\mathrm{U} 12$ & $\mathrm{U} 13$ & $\mathrm{U} 23$ \\
\hline $\mathrm{S}(1)$ & $0.0221(7)$ & $0.0409(10)$ & $0.0287(9)$ & $0.0002(6)$ & $0.0039(8)$ & $-0.0080(9)$ \\
$\mathrm{O}(1)$ & $0.035(2)$ & $0.040(3)$ & $0.036(2)$ & $0.021(2)$ & $-0.003(2)$ & $-0.008(2)$ \\
$\mathrm{O}(2)$ & $0.020(2)$ & $0.024(3)$ & $0.044(3)$ & $0.006(2)$ & $-0.001(2)$ & $0.001(2)$ \\
$\mathrm{N}(1)$ & $0.017(2)$ & $0.031(3)$ & $0.016(3)$ & $0.004(3)$ & $0.007(2)$ & $-0.007(2)$ \\
$\mathrm{C}(1)$ & $0.037(4)$ & $0.024(3)$ & $0.018(3)$ & $-0.002(4)$ & $-0.004(3)$ & $0.003(3)$ \\
$\mathrm{C}(2)$ & $0.047(4)$ & $0.047(4)$ & $0.031(4)$ & $-0.011(4)$ & $0.006(3)$ & $-0.001(3)$ \\
$\mathrm{C}(3)$ & $0.029(4)$ & $0.032(4)$ & $0.031(4)$ & $-0.002(3)$ & $-0.007(3)$ & $0.001(3)$ \\
$\mathrm{C}(4)$ & $0.045(4)$ & $0.028(4)$ & $0.039(4)$ & $0.000(4)$ & $0.009(4)$ & $0.001(3)$ \\
$\mathrm{C}(5)$ & $0.014(3)$ & $0.035(4)$ & $0.022(3)$ & $0.003(3)$ & $0.004(3)$ & $-0.006(3)$ \\
$\mathrm{C}(6)$ & $0.009(3)$ & $0.031(4)$ & $0.028(3)$ & $0.000(3)$ & $-0.007(3)$ & $-0.007(3)$ \\
$\mathrm{C}(7)$ & $0.015(4)$ & $0.033(4)$ & $0.031(4)$ & $-0.002(3)$ & $-0.005(3)$ & $0.001(3)$ \\
$\mathrm{C}(8)$ & $0.027(3)$ & $0.030(4)$ & $0.054(4)$ & $0.002(3)$ & $-0.002(4)$ & $0.007(3)$ \\
$\mathrm{C}(9)$ & $0.050(5)$ & $0.039(4)$ & $0.091(6)$ & $-0.006(4)$ & $-0.007(5)$ & $0.007(4)$ \\
$\mathrm{C}(10)$ & $0.025(4)$ & $0.021(4)$ & $0.036(4)$ & $-0.003(3)$ & $-0.003(3)$ & $-0.012(3)$ \\
$\mathrm{C}(11)$ & $0.054(4)$ & $0.027(4)$ & $0.069(5)$ & $-0.004(4)$ & $0.014(4)$ & $-0.005(4)$ \\
$\mathrm{C}(12)$ & $0.052(4)$ & $0.026(4)$ & $0.038(4)$ & $0.017(3)$ & $0.010(4)$ & $0.000(3)$ \\
$\mathrm{C}(13)$ & $0.066(4)$ & $0.032(4)$ & $0.054(4)$ & $0.003(4)$ & $-0.022(5)$ & $-0.018(4)$ \\
\hline
\end{tabular}


Table S4. Bond Lengths( $(\AA)$ for anti-5h

\begin{tabular}{|c|c|c|c|c|c|}
\hline$\overline{\text { atom }}$ & atom & distance & atom & atom & distance \\
\hline S1 & $\mathrm{O} 1$ & $1.508(3)$ & $\mathrm{C} 5$ & C6 & $1.524(7)$ \\
\hline S1 & N1 & $1.659(4)$ & $\mathrm{C} 5$ & $\mathrm{C} 10$ & $1.561(7)$ \\
\hline S1 & $\mathrm{C} 1$ & $1.835(5)$ & C6 & $\mathrm{C} 7$ & $1.509(7)$ \\
\hline $\mathrm{O} 2$ & $\mathrm{C} 7$ & $1.438(6)$ & $\mathrm{C} 7$ & $\mathrm{C} 8$ & $1.515(7)$ \\
\hline N1 & $\mathrm{C} 5$ & $1.465(6)$ & $\mathrm{C} 8$ & C9 & $1.525(8)$ \\
\hline $\mathrm{C} 1$ & $\mathrm{C} 2$ & $1.518(7)$ & $\mathrm{C} 10$ & $\mathrm{C} 11$ & $1.506(8)$ \\
\hline $\mathrm{C} 1$ & $\mathrm{C} 3$ & $1.512(7)$ & $\mathrm{C} 10$ & $\mathrm{C} 12$ & $1.529(8)$ \\
\hline $\mathrm{C} 1$ & $\mathrm{C} 4$ & $1.531(8)$ & $\mathrm{C} 10$ & $\mathrm{C} 13$ & $1.528(8)$ \\
\hline
\end{tabular}

Table S5. Bond Angles( $\left({ }^{\circ}\right)$ for anti-5h

\begin{tabular}{llllllll}
\hline atom & atom & atom & angle & atom & atom & atom & angle \\
\hline O1 & S1 & N1 & $112.0(2)$ & C6 & C5 & C10 & $114.7(4)$ \\
O1 & S1 & C1 & $104.4(2)$ & C5 & C6 & C7 & $115.7(4)$ \\
N1 & S1 & C1 & $99.7(2)$ & C2 & C7 & C6 & $111.9(4)$ \\
S1 & N1 & C5 & $115.9(3)$ & C2 & C7 & C8 & $107.9(5)$ \\
S1 & C1 & C2 & $104.4(4)$ & C6 & C7 & C8 & $111.3(5)$ \\
S1 & C1 & C3 & $109.7(4)$ & C7 & C8 & C9 & $114.4(5)$ \\
S1 & C1 & C4 & $108.8(4)$ & C5 & C10 & C11 & $108.5(4)$ \\
C2 & C1 & C3 & $110.5(5)$ & C5 & C10 & C12 & $110.4(4)$ \\
C2 & C1 & C4 & $110.8(5)$ & C5 & C10 & C13 & $109.0(5)$ \\
C3 & C1 & C4 & $112.4(5)$ & C11 & C10 & C12 & $109.3(5)$ \\
N1 & C5 & C6 & $111.0(4)$ & C11 & C10 & C13 & $110.3(5)$ \\
N1 & C5 & C10 & $110.4(4)$ & C12 & C10 & C13 & $109.4(5)$ \\
\hline
\end{tabular}


Table S6. Torsion Angles $\left({ }^{\circ}\right)$ for anti-5h

\begin{tabular}{llllllllll}
\hline atom & atom & atom & atom & angle & atom & atom & atom & atom & angle \\
\hline S1 & N1 & C5 & C6 & $115.9(4)$ & N1 & C5 & C6 & C7 & $-53.4(6)$ \\
S1 & N1 & C5 & C10 & $-115.6(4)$ & N1 & C5 & C10 & C11 & $62.6(5)$ \\
O1 & S1 & N1 & C5 & $-79.0(4)$ & N1 & C5 & C10 & C12 & $-57.1(6)$ \\
O1 & S1 & C1 & C2 & $75.1(4)$ & N1 & C5 & C10 & C13 & $-177.3(5)$ \\
O1 & S1 & C1 & C3 & $-43.3(4)$ & C1 & S1 & N1 & C5 & $171.1(4)$ \\
O1 & S1 & C1 & C4 & $-166.6(4)$ & C5 & C6 & C7 & C8 & $-173.9(5)$ \\
O2 & C7 & C6 & C5 & $-53.1(6)$ & C6 & C5 & C10 & C11 & $-171.0(5)$ \\
O2 & C7 & C8 & C9 & $62.1(6)$ & C6 & C5 & C10 & C12 & $69.3(6)$ \\
N1 & S1 & C1 & C2 & $-169.1(4)$ & C6 & C5 & C10 & C13 & $-50.9(6)$ \\
N1 & S1 & C1 & C3 & $72.5(4)$ & C6 & C7 & C8 & C9 & $-174.8(4)$ \\
N1 & S1 & C1 & C4 & $-50.8(4)$ & C7 & C6 & C5 & C10 & $-179.5(4)$ \\
\hline
\end{tabular}

Table S7. Non-Bonded Contacts out to 3.60 Å for anti-5h

\begin{tabular}{llllllll}
\hline atom & atom & distance & ADC & atom & atom & distance & ADC \\
\hline $\mathrm{O} 1$ & $\mathrm{C} 4$ & $3.491(7)$ & 4 & $\mathrm{O} 1$ & $\mathrm{O} 2$ & $2.780(5)$ & 1 \\
$\mathrm{O} 2$ & $\mathrm{~N} 1$ & $3.066(6)$ & 45503 & & & & \\
\hline
\end{tabular}




\section{References}

(1) Still, W. C.; Kahn, M.; Mitra, A. J. Org. Chem. 1978, 43, 2923-2925.

(2) Liu, G.; Cogan, D. A.; Owens, T. D.; Tang, T. P.; Ellman, J. A. J. Org. Chem. 1999, 64, 1278-1284.

(3) Grigg, R.; Markandu, J.; Perrior, T.; Surendrakumar, S.; Warnock, W. J. Tetrahedron 1992, 48, 6929-6952.

(4) Loh, T.-P.; Li, X.-R. Tetrahedron 1999, 55, 10789-10802.

(5) Andres, J. M.; Pedrosa, R.; Perez-Encabo, A. Tetrahedron 2000, 56, 1217-1223.

(6) Denmark, S. E.; Stavenger, R. A. J. Am. Chem. Soc. 2000, 122, 8837-8847.

(7) Mill, S.; Hootele, C. J. Nat. Prod. 2000, 63, 762-764.

(8) Michel, K.-H.; Sandberg, F.; Haglid, F.; Norin, T. Acta. Pharm. Suec. 1967, 4, 97-116. 